\title{
RESONANCES OF 4-TH ORDER DIFFERENTIAL OPERATORS
}

\author{
ANDREY BADANIN AND EVGENY L. KOROTYAEV
}

\begin{abstract}
We consider fourth order ordinary differential operator with compactly supported coefficients on the line. We define resonances as zeros of the Fredholm determinant which is analytic on a four sheeted Riemann surface. We determine asymptotics of the number of resonances in complex discs at large radius. We consider resonances of an Euler-Bernoulli operator on the real line with the positive coefficients which are constants outside some finite interval. We show that the Euler-Bernoulli operator has no eigenvalues and resonances iff the positive coefficients are constants on the whole axis.
\end{abstract}

\section{INTRODUCTION AND MAIN RESULTS}

1.1. Introduction. There are a lot of results about resonances for 1-dim second order operators, see e.g., [F97], [H99], [K04], [S00], [Z87] and references therein. We can say that problems of resonances for these operators are well understood. The resonance scattering for third order operators on the line was considered in [K16]. There are a lot of papers [B85], [I88], [Iw88], .... and even a book [BDT88] about scattering for one dimensional higher order $\geqslant 3$ operators. Unfortunately, even the inverse scattering problems for higher order operators on the line are not solved and there are few results about resonances [K16], BK17].

We discuss resonances of fourth order differential operators $H$ with compactly supported coefficients on the line given by

$$
H=H_{0}+V, \quad V=2 \partial p \partial+q, \quad \partial=\frac{d}{d x} .
$$

where the operator $H_{0}=\partial^{4}$ is unperturbed. Below we assume that our coefficients $p, q \in \mathcal{H}_{0}$, where $\mathcal{H}_{m}, m=0,1,2, \ldots$, are the spaces of compactly supported functions

$$
\mathcal{H}_{m}=\mathcal{H}_{m}(\gamma)=\left\{f \in L^{1}(\mathbb{R}): \operatorname{supp} f \subset[0, \gamma], f^{(m)} \in L^{1}(0, \gamma)\right\}
$$

for some $\gamma>0$. Our operator $H$ is self-adjoint on the corresponding form domain (see Sect. 2). The operator $H$ has purely absolutely continuous spectrum $[0, \infty)$ plus a finite number of simple eigenvalues on the real line, see Theorem 1.1.

We define the Fredholm determinant by (1.5), which is analytic on a four sheeted Riemann surface of the function $\lambda^{\frac{1}{4}}$. We define a resonance of the operator $H$ as a zero of the Fredholm determinant on the non-physical sheets of this surface.

Our main goal is to obtain estimates of $D$ and determine asymptotics of the number of resonances in the large disc. Moreover, we derive trace formulas in terms of resonances and prove a Borg type results about resonances for Euler-Bernoulli operator.

\footnotetext{
Date: August 29, 2018.

1991 Mathematics Subject Classification. 34L25 (47E05 47N50).

Key words and phrases. fourth order operators, resonances, scattering, trace formula.
} 


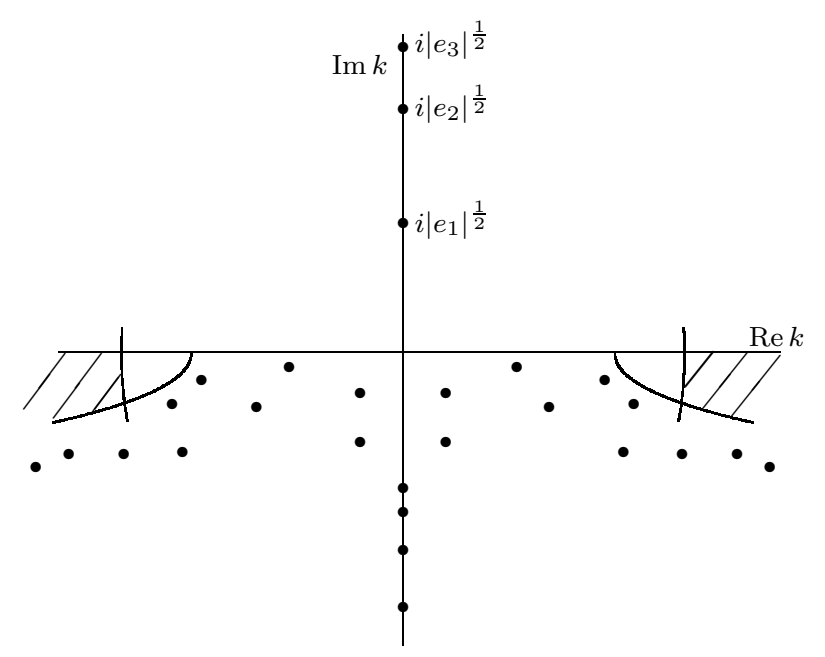

FigURE 1. Resonances for the Schrödinger operator $h$. The resonances are marked by circles. The forbidden domain for the resonances is shaded.

1.2. Schrödinger operator. In order to discuss resonances for fourth order operators we consider a Schrödinger operator $h$ on $L^{2}(\mathbb{R})$ given by

$$
h=h_{0}-p, \quad h_{0}=-\frac{d^{2}}{d x^{2}},
$$

where $h_{0}$ is the unperturbed operator and the compactly supported potential $p \in \mathcal{H}_{0}$. We recall the well known results for the operator $h$, see, e.g., [DT79], [Fa64]. The operator $h$ has purely absolutely continuous spectrum $[0, \infty)$ plus a finite number of simple eigenvalues $e_{1}<e_{2}<\ldots<e_{N}<0$.

We define the resolvent $r_{0}(k)$ and the operator $y_{0}(k)$ for $k \in \mathbb{C}_{+}$by

$$
r_{0}(k)=\left(h_{0}-k^{2}\right)^{-1}, \quad y_{0}(k)=|p|^{\frac{1}{2}} r_{0}(k) p^{\frac{1}{2}}, \quad \text { where } \quad p^{\frac{1}{2}}=|p|^{\frac{1}{2}} \operatorname{sign} p .
$$

Each operator $y_{0}(k), k \in \mathbb{C}_{+}$, is trace class and thus we can introduce the Fredholm determinant by

$$
d(k)=\operatorname{det}\left(I+y_{0}(k)\right), \quad k \in \mathbb{C}_{+} .
$$

The function $d(k)$ is analytic in $\mathbb{C}_{+}$and has an analytic extension onto the whole complex plane without zero. It has simple zeros (the eigenvalues) $i\left|e_{1}\right|^{\frac{1}{2}}, \ldots, i\left|e_{N}\right|^{\frac{1}{2}} \in i \mathbb{R}_{+}$in $\mathbb{C}_{+}$and there are no other zeros in $\mathbb{C}_{+}$. Moreover, $d(k)$ has an infinite number of zeros (resonances) in $\mathbb{C}_{-}$. The operator $h$ is self-adjoint and then the set of resonances is symmetric with respect to the imaginary axis, see Fig:1.

The problems of resonances for 1-dimensional Schrödinger operators with compactly supported potentials are well understood. Recall the following results:

- Let $\gamma=\sup (\operatorname{supp}(p))$ and let $n(r)$ be the number of zeros of $d(k)$ in a disk $|k|<r$. Zworski [Z87] determined the following asymptotics (see also [F97], [K05], [S00])

$$
n(r)=\frac{2 \gamma r}{\pi}+o(r) \quad \text { as } \quad r \rightarrow \infty
$$

For each $\delta>0$ the number of zeros of $d(k)$ with modulus $\leqslant r$ lying outside both of the two sectors $|\arg k|,|\arg k-\pi|<\delta$ is $o(r)$ for large $r$.

- There are only finitely many resonances in the domain $\left\{k \in \mathbb{C}_{-}:|k|>\|p\| e^{\|p\|} e^{-2 \gamma \operatorname{Im} k}\right\}$. 


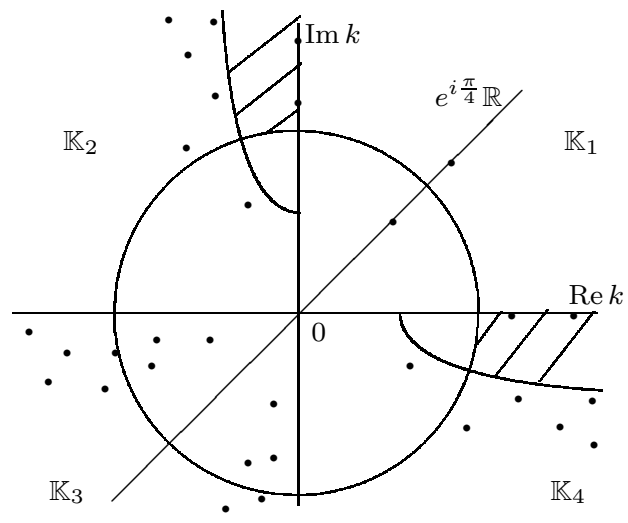

Figure 2. The plane of variable $k$. The function $D(k)$ is real on the line $e^{i \frac{\pi}{4}} \mathbb{R}$. The resonances are marked by circles. The forbidden domain for the resonances is shaded.

- The resonances may have any multiplicity (see [K05]).

- Inverse resonance problem (uniqueness, characterization and recovering) was solved in terms of resonances for the Schrödinger operator with a compactly supported potential on the real line [K05].

- Stability estimates for resonances were determined in [K04x] and [MSW10].

- Lieb-Thirring type inequalities for resonances were obtained in [K16xx].

1.3. Determinant. In order to define the Fredholm determinant for the operator $H$ we need a factorization of the perturbation $V$ in the form

$$
V=V_{1} V_{2}, \quad V_{1}=\left(\partial|2 p|^{\frac{1}{2}},|q|^{\frac{1}{2}}\right), \quad V_{2}=\left(\begin{array}{c}
(2 p)^{\frac{1}{2}} \partial \\
q^{\frac{1}{2}}
\end{array}\right) .
$$

We introduce a new spectral variable $k \in \mathbb{K}_{1}$ by $\lambda=k^{4}$, where the spectral parameter $\lambda$ belongs to the cut plane $\mathbb{C} \backslash\left[0, \infty\right.$ ) (see Fig 2 ) and the sector $\mathbb{K}_{1}$ is given by

$$
\mathbb{K}_{1}=\left\{k \in \mathbb{C}: \arg k \in\left(0, \frac{\pi}{2}\right)\right\} .
$$

Introduce the free resolvent $R_{0}(k)$ and the operator $Y_{0}(k)$ by

$$
R_{0}(k)=\left(H_{0}-k^{4}\right)^{-1}, \quad Y_{0}(k)=V_{2} R_{0}(k) V_{1}, \quad k \in \mathbb{K}_{1} .
$$

In Proposition 1.1 we show that each operator $Y_{0}(k), k \in \mathbb{K}_{1}$, is trace class. Thus we can define the Fredholm determinant $D(k), k \in \mathbb{K}_{1}$, by

$$
D(k)=\operatorname{det}\left(I+Y_{0}(k)\right) \text {. }
$$

If $k \in \overline{\mathbb{K}}_{1} \backslash\{0\}$ is a zero of the determinant $D$, then $\lambda=k^{4} \in \mathbb{R} \backslash\{0\}$ and $\lambda$ is an eigenvalue of the operator $H$. We present preliminary results about the determinant.

Proposition 1.1. Let $p, q \in \mathcal{H}_{0}$. Then

i) Each operator $Y_{0}(k), k \in \mathbb{K}_{1}$, is trace class and the operator-valued function $k^{3} Y_{0}(k)$ is entire in the trace norm.

ii) The Fredholm determinant $D(k)$ is analytic in $\mathbb{K}_{1}$ and has an analytic extension into the whole plane without zero such that the function $k^{4} D(k)$ is entire. In particular, the operator 
$H$ has a finite number of eigenvalues. Moreover, $D(k)$ is real on the line $e^{i \frac{\pi}{4}} \mathbb{R}$ and satisfies:

$$
\begin{gathered}
D(k)=\overline{D(i \bar{k})} \quad \forall k \neq 0, \\
D(k)=1-\frac{1+i}{2 k} \int_{\mathbb{R}} p(x) d x+\frac{O(1)}{k^{2}} \quad \text { as } \quad|k| \rightarrow \infty, \quad k \in \overline{\mathbb{K}}_{1},
\end{gathered}
$$

uniformly in $\arg k \in\left[0, \frac{\pi}{2}\right]$.

Remark. The function $D(k)$ is symmetric with respect to the line $e^{i \frac{\pi}{4}} \mathbb{R}$. Thus it is sufficiently to analyze this function in the half-plane $e^{i \frac{\pi}{4}} \mathbb{C}_{+}$.

The zeros of the function $D$ in $\mathbb{C} \backslash \overline{\mathbb{K}}_{1}$ are called resonances of $H$. Let $\mathcal{N}(r)$ be the number of zeros of the function $D$ in the disc $|k|<r$, counted with multiplicity. Introduce the domains

$$
\mathbb{K}_{j}=i^{j-1} \mathbb{K}_{1}, \quad j=1,2,3,4 \text {. }
$$

Now we formulate our first main result.

Theorem 1.2. Let $p, q \in \mathcal{H}_{0}$. Then the determinant $D(k)$ and the counting function $\mathcal{N}(r)$ satisfy

$$
\begin{gathered}
|D(k)| \leqslant C e^{2 \gamma\left((\operatorname{Re} k)_{-}+(\operatorname{Im} k)_{-}\right)}, \quad \forall \quad k \in \mathbb{C}, \quad|k| \geqslant 1, \\
\mathcal{N}(r) \leqslant \frac{4 \gamma r}{\pi}(1+o(1)) \quad \text { as } \quad r \rightarrow \infty,
\end{gathered}
$$

where $(a)_{-}=\frac{|a|-a}{2}$. Moreover, if $k_{*} \in \mathbb{K}_{2}$ is a resonance, then

$$
\left|k_{*}\right| \leqslant C e^{-2 \gamma \operatorname{Re} k_{*}} \text {. }
$$

Here $C=C(p, q)>0$ is some constant depending on $p, q$ only.

Remark. 1) The estimate (1.10) gives that the domain $\left\{k \in \mathbb{K}_{2}:|k|>C e^{-2 \gamma \operatorname{Re} k}\right\}$ is forbidden for resonances in $\mathbb{K}_{2}$ and, due to the symmetry of the function $D(k)$, the domain $\left\{k \in \mathbb{K}_{4}:|k|>C e^{-2 \gamma \operatorname{Im} k}\right\}$ is forbidden for resonances in $\mathbb{K}_{4}$, see Fig. 2. The proof repeats arguments from [K04].

2) Estimate (1.8) is crucial to prove trace formula (1.16) in terms of resonances.

3) Due to (1.6) the set of resonances is symmetric with respect to the line $e^{i \frac{\pi}{4}}$ (with the same multiplicity).

We describe the main difference between the case of the second and fourth order operators.

- Recall that for Schrödinger operators the Riemann surface in terms of the momentum $k$ is the complex plane. The upper half-plane corresponds to the physical sheet and the lower half-plane corresponds to the non-physical sheet. In order to determine asymptotics of the determinant $d(k)$ of the Schrödinger operator in the complex plane it is sufficiently to obtain the asymptotics of the determinant and the $2 \times 2$ scattering matrix in $\mathbb{C}_{+}$. The Birman-Krein formula (see $(2.10)$ ) gives the asymptotics of the determinant in $\mathbb{C}_{-}$.

- In the case of fourth order operators the Riemann surface has 4 sheets and each quadrant $\mathbb{K}_{j} \subset \mathbb{C}, j=1,2,3,4$ of the variable $k=\lambda^{\frac{1}{4}}$ corresponds to the sheet $\Lambda_{j}$ of the Riemann surface $\lambda^{\frac{1}{4}}$. Using arguments similar to the second order case we obtain asymptotics of the determinant in the domains $\mathbb{K}_{1}, \mathbb{K}_{2}$ only (and, by the symmetry, in $\mathbb{K}_{4}$ ). In fact, if we have asymptotics of the determinant $D(k)$ and asymptotics of the $2 \times 2$ scattering matrix $S(k)$ in $\mathbb{K}_{1}$, then using the identity (4.9) we obtain the asymptotics of the determinant $D(k)$ in $\mathbb{K}_{2}$. In order to obtain the asymptotics in the domain $\mathbb{K}_{3}$ we use additional arguments from 
[K16] (for third order operators), more complicated than for the sectors $\mathbb{K}_{1}, \mathbb{K}_{2}$. We need to introduce an additional $4 \times 4$ matrix-valued function $\Omega(k)$, which satisfies the identity (4.10). Having asymptotics of the determinant $D(k)$ and of this $4 \times 4$ matrix $\Omega(k)$ in $\mathbb{K}_{1}$ and using the identity (4.10) we obtain the asymptotics of the determinant $D(k)$ in $\mathbb{K}_{3}$. Note that in order to determine asymptotics of determinants for $N$ order operators we need to introduce the corresponding $N \times N$ matrix-valued functions.

1.4. Asymptotics of resonances. Introduce the Cartwright class of functions. Recall that an entire function $f(z)$ is said to be of exponential type if there is a constant $A$ such that $|f(z)| \leqslant$ const $e^{A|z|}$ everywhere. The infimum of the set of $A$ for which such inequality holds is called the type of $f$. For each exponential type function $f$ we define the types $\rho_{ \pm}(f)$ in $\mathbb{C}_{ \pm}$ by

$$
\rho_{ \pm}(f) \equiv \lim \sup _{y \rightarrow \infty} \frac{\log |f( \pm i y)|}{y} .
$$

The function $f$ is said to belong to the Cartwright class $\mathcal{C}_{\text {Cart }}$ if $f$ is entire, of exponential type, and satisfies the following conditions:

$$
\int_{\mathbb{R}} \frac{\log (1+|f(x)|)}{1+x^{2}} d x<\infty, \quad \rho_{+}(f)=0, \quad \rho_{-}(f)=2 \rho>0,
$$

for some $\rho>0$. We recall the Levinson Theorem (see [Ko88]):

Let an entire function $f$ be in the Cartwright class $\mathcal{C}_{\text {Cart }}$. Let $\mathcal{N}(r, f)$ be the number of zeros of the function $f$ in the disc $|k|<r$, counted with multiplicity. Then

$$
\mathcal{N}(r, f)=\frac{2 \rho}{\pi} r+o(r) \quad \text { as } \quad r \rightarrow \infty .
$$

Roughly speaking the Fredholm determinants of second order operators on the real line with compactly supported potential belong to the Cartwright class:

- Schrödinger operators with compactly supported potentials [Z87],

- Schrödinger operators with periodic plus compactly supported potentials [K11,

- Schrödinger operators with matrix-valued compactly supported potentials [N07],

- Dirac operators with $2 \times 2$ matrix-valued compactly supported potentials [IK14].

In all these cases the Fredholm determinants are entire and belong to the Cartwright class, and the corresponding Riemann surfaces are two sheeted. Thus the Levinson Theorem describes the distribution of resonances in the large disc.

We underline that the Fredholm determinants of the Stark operator with compactly supported potential does not belong to the Cartwright class, since the order of determinants is $\frac{3}{2}$, see $[\mathrm{K} 16 \mathrm{x}$. In this paper we show that the determinant for a fourth order operators does not belong to the Cartwright class also.

We determine asymptotics of resonances for fourth order operators under the stronger conditions for the coefficient $p$ and the standard one for $q$ :

$$
(p, q) \in \mathcal{H}_{1} \times \mathcal{H}_{0}, \quad p_{+} p_{-} \neq 0, \quad \text { where } \quad p_{-}=p(+0), \quad p_{+}=p(\gamma-0) .
$$

Introduce the model numbers $k_{n}^{0}, n \in \pm \mathbb{N}$ (see Fig. 3) by

$$
k_{n}^{0}=\frac{1}{\gamma}\left(i \pi j_{n}-2 \log \frac{4 \pi n}{\gamma\left|p_{+} p_{-}\right|^{\frac{1}{4}}}\right), \quad k_{-n}^{0}=i k_{n}^{0}, \quad n \in \mathbb{N},
$$




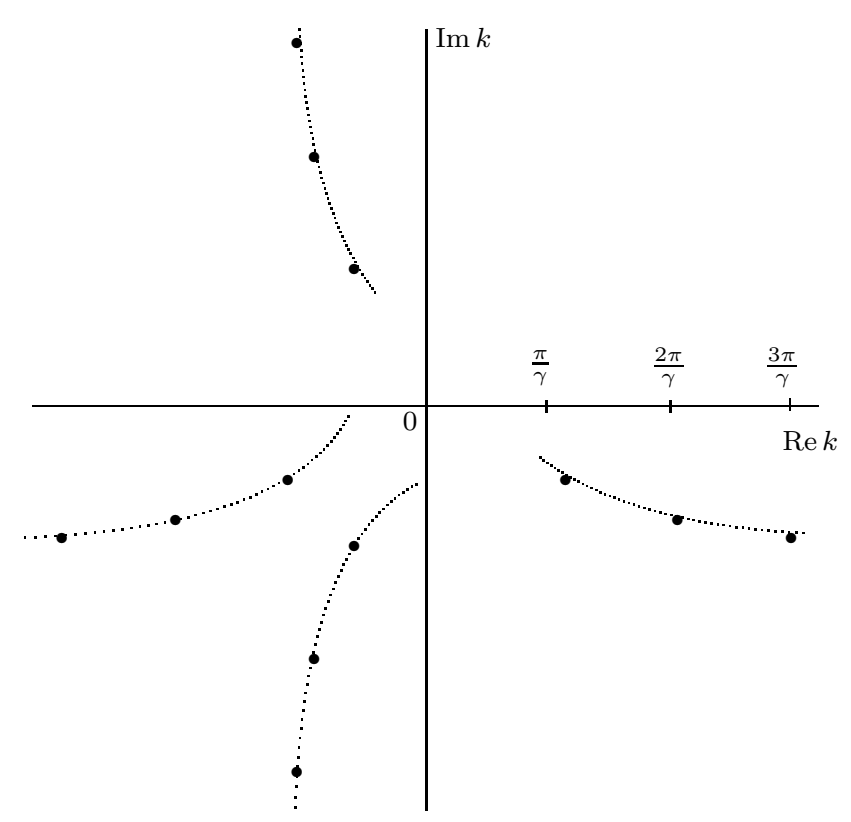

Figure 3. Asymptotics of resonances for step coefficients

where

$$
j_{n}=\left\{\begin{array}{ll}
n, & \text { if } p_{+} p_{-}>0 \\
n+\frac{1}{2}, & \text { if } p_{+} p_{-}<0
\end{array} .\right.
$$

Theorem 1.3. Let $p, q$ satisfy the conditions (1.12). Then for any $\varepsilon \in\left(0, \frac{\pi}{2 \gamma}\right)$ there exists $\rho>0$ such that in each disk $\left\{\left|k-k_{n}^{0}\right|<\varepsilon\right\} \subset e^{i \frac{\pi}{4}} \mathbb{C}_{+} \cap\{|k|>\rho\}, n \in \pm \mathbb{N}$, there exists exactly one resonance $k_{n}$ and there are no other resonances in the domain $e^{i \frac{\pi}{4}} \mathbb{C}_{+} \cap\{|k|>\rho\}$. These resonances satisfy

$$
k_{n}=k_{n}^{0}+o(1) \quad \text { as } \quad n \rightarrow \pm \infty .
$$

In particular, there are finitely many zeros of $D(k)$ on $\mathbb{R} \cup i \mathbb{R}$. Moreover, let $\mathcal{N}_{j}(r), j=2,3,4$ be the number of zeros of the function $D$ in a domain $\mathbb{K}_{j} \cap\{|k|<r\}$ counted with multiplicity. Then

$$
\begin{gathered}
\mathcal{N}_{2}(r)=\mathcal{N}_{4}(r)=\frac{\gamma r}{\pi}(1+o(1)), \\
\mathcal{N}_{3}(r)=\frac{2 \gamma r}{\pi}(1+o(1))
\end{gathered}
$$

as $r \rightarrow \infty$ and the determinant $D$ does not belong to the Cartwright class.

Remark. From (1.14) we obtain $\mathcal{N}_{3}(r)=2 \mathcal{N}_{2}(r)(1+o(1))$. It means that the number of resonances in the large disc in the domain $\mathbb{K}_{3}$ is in two times more than in the domain $\mathbb{K}_{2}$, see Fig. 4 .

Our next results are devoted to trace formulas in terms of resonances. Recall that the function $D(k)$ may have a pole at the point $k=0$. Then the function $D(k)$ satisfies

$$
D(k)=\frac{\alpha}{k^{m}}\left(1+\beta k+O\left(k^{2}\right)\right) \quad \text { as } \quad|k| \rightarrow 0
$$



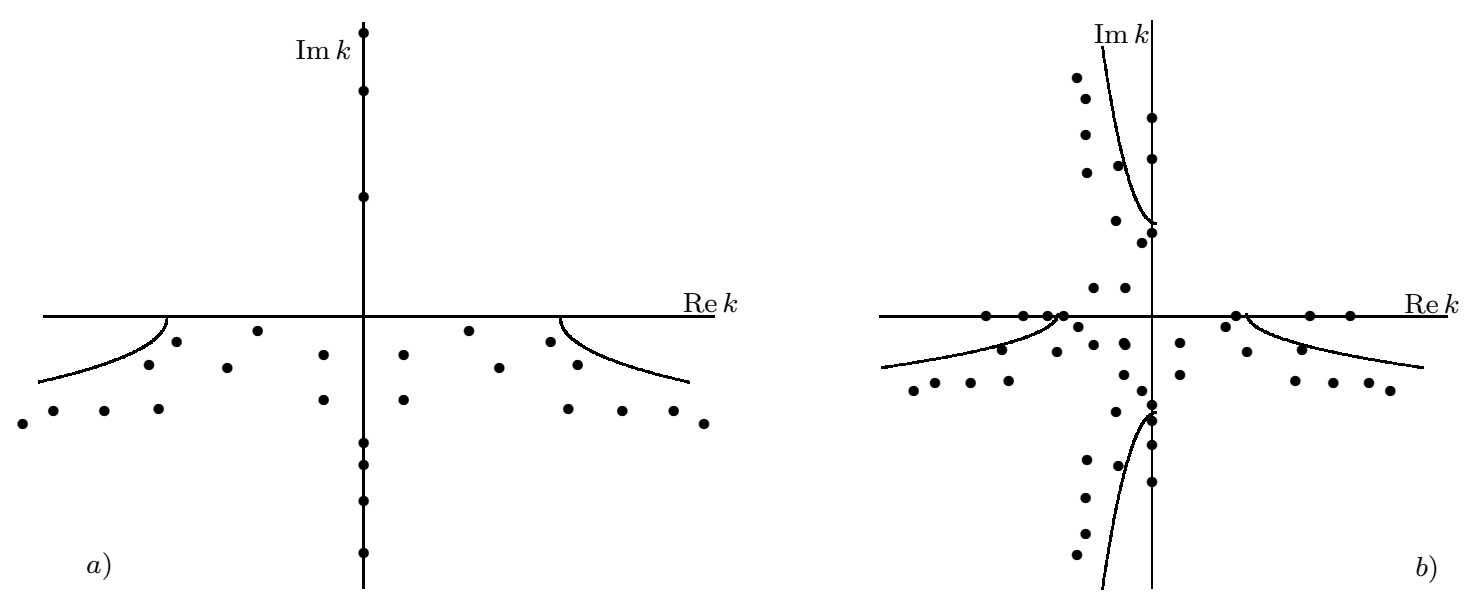

FIGURE 4. a) Resonances for the second order operator $h$; b) Resonances for the fourth order operator $h^{2}$.

for some $\alpha, \beta \in \mathbb{C}$, where $m \leqslant 4$ is an order of the pole. Let $\zeta_{n}, n \in \mathbb{N}$, be the zeros of the function $D$ in $\mathbb{C} \backslash\{0\}$ labeled by $0<\left|\zeta_{1}\right| \leqslant\left|\zeta_{2}\right| \leqslant \ldots$ counting with multiplicities.

Theorem 1.4. Let $p, q \in \mathcal{H}_{0}$ and let $R(k)=\left(H-k^{4}\right)^{-1}$. Then the following trace formula

$$
\operatorname{Tr}\left(R_{0}(k)-R(k)\right)=\frac{1}{4 k^{3}}\left(\beta-\frac{m}{k}+k \lim _{r \rightarrow \infty} \sum_{\left|\zeta_{n}\right|<r} \frac{1}{\zeta_{n}\left(k-\zeta_{n}\right)}\right)
$$

holds true for all $k \in \mathbb{C} \backslash\left\{0, \zeta_{n}, n \in \mathbb{N}\right\}$, where the series converges absolutely and uniformly on any compact subset in $\mathbb{C} \backslash\left\{0, \zeta_{n}, n \in \mathbb{N}\right\}$, and $\beta, m$ are defined in (1.15).

Remark. 1) In the proof we use arguments from [K04], [K16].

2) A trace formula for the scattering phase function is proved in Theorem 5.2 .

1.5. Euler-Bernoulli operators. We discuss resonances of the Euler-Bernoulli operator

$$
\mathcal{E} u=\frac{1}{b}\left(a u^{\prime \prime}\right)^{\prime \prime},
$$

acting on $L^{2}(\mathbb{R}, b(x) d x)$. We assume that the coefficients $a, b$ are positive, $a=b=1$ outside a unit interval and satisfy $a-1, b-1 \in \mathcal{H}_{4}(1)$. The Euler-Bernoulli operator describes the relationship between the thin beam's deflection and the applied load, $a$ is the rigidity and $b$ is the density of the beam, see, e.g., [TW59].

In Sect 5.3 we show that the operator $\mathcal{E} \geqslant 0$ is unitarily equivalent to an operator $H \geqslant 0$ with specific coefficients $p, q \in \mathcal{H}_{0}$. Then we can define a determinant for the operator $\mathcal{E}$ as the determinant for the operator $H$ with these $p, q$. Applying the results for the operator $H$ to the operator $\mathcal{E}$ we obtain the following corollary of Theorem 1.2 .

Corollary 1.5. Let $a-1$ and $b-1 \in \mathcal{H}_{4}(1)$ and let $a, b$ be positive. Then the determinant $D(k)$, the counting function $\mathcal{N}(r)$ and the resonances for the Euler-Bernoulli operator $\mathcal{E}$ satisfy the estimates (1.8)-(1.10), where

$$
\gamma=\int_{0}^{1}\left(\frac{b(x)}{a(x)}\right)^{\frac{1}{4}} d x
$$


Now we formulate our result about the inverse resonance scattering for the Euler-Bernoulli operator $\mathcal{E}$.

Theorem 1.6. Let $a-1, b-1 \in \mathcal{H}_{4}$ and let $a, b$ be positive. Then the operator $\mathcal{E}$ does not have any eigenvalues and resonances iff $a=b=1$ on the whole line.

1.6. Historical review. There are a lot of results about resonances. We recall that resonances, from a physicists point of view, were first studied by Gamov [Ga28]. Since then, properties of resonances have been the object of intense study and we refer to [SZ91 for the mathematical approach in the multi-dimensional case and references given therein. We discuss the one-dimensional case. A lot of papers are devoted to the resonances the one-dimensional Schrödinger operators with compactly supported potentials, see Froese [F97], Korotyaev [K04], Simon [S00], Zworski [Z87] and references given there. We recall that Zworski [Z87] obtained the first results about the asymptotic distribution of resonances for the Schrödinger operator with compactly supported potentials on the real line. Inverse problems (characterization, recovering, plus uniqueness) in terms of resonances were solved by Korotyaev for the Schrödinger operator with a compactly supported potential on the real line [K05] and the half-line [K04], see also Zworski [Z02] concerning the uniqueness.

There are few papers devoted to systems. Nedelec [N07] considered resonances for Schrödinger operators with compactly supported matrix-valued potentials on the real line. Iantchenko and Korotyaev [IK14 considered the Dirac operator on the real line with 2x2 matrixvalued compactly supported potentials. They obtained asymptotics of counting function of resonances, estimates on the resonances and the forbidden domain, a trace formula in terms of resonances. Lieb-Thirring type inequality for resonances of Dirac operators with compactly supported matrix-valued potentials on the real line is obtained in [K14]. Resonances for Stark operators on the real line are considered in $[\mathrm{K} 16 \mathrm{x}$. Here we underline that for all these cases the corresponding Riemann surfaces are two-sheeted similar to the Schrödinger operator case.

A lot of papers are devoted to the inverse scattering theory for fourth order operators on the line, see papers Aktosun and Papanicolaou [AP08, Butler [Bu68], Iwasaki [I88], [Iw88], Hoppe, Laptev and Östensson [HLO06] and the book Beals, Deift, Tomei [BDT88] and references therein.

Resonances for higher order operators with compactly supported coefficients were considered by Korotyaev [K16] firstly for the case of third order operators. Here general properties of resonances were described. In particular, upper bounds of the number of resonances in complex discs at large radius and the trace formula in terms of resonances were obtained. Note that this case is very complicated for the consideration since the Born term roughly speaking is constant. Recall that for Schrödinger operators the corresponding Born term is the Fourier transformation of the potential. It is important for the global analysis of resonances, including inverse problems.

Resonances of fourth order operators with compactly supported coefficients on the halfline were studied by Badanin and Korotyaev BK17. Asymptotics of resonances and trace formulas in terms of resonances were determined. This case is simpler, than the case of the line considered in the present paper, because the scattering matrix is a scalar function. An extension of the determinant onto the third quadrant $\mathbb{K}_{3}$ may be obtained using a $2 \times 2$ matrix-valued function $\Omega$. Here the technique from [K16] was used. But it is important that the corresponding Born term is expressed in terms of the Fourier transformations of the compactly supported coefficients. 
In the present paper the corresponding matrix $\Omega$ for an operator on the line is a $4 \times 4$ matrixvalued function and there are some algebraic difficulties in order to obtain this extension. Clearly, the problem for higher order operators will be much more complicated, especially for odd order case.

The usual applications of fourth order differential operators are bending vibrations of thin beams and plates described by the Euler-Bernoulli equation. Many problems of engineering involve solutions of scattering problem for the Euler-Bernoulli equation, see [Gr75] and references therein. Furthermore, the inverse spectral problem methods for some non-linear partial differential equations lead to fourth order operators, see [HLO06].

The plan of the paper is as follows. In Section 2 we study properties of the resolvent of the operator $H_{0}$. In Sections 3 and 4 we consider the scattering matrix and the determinant. In Sections 5 we prove Proposition 1.1 and Theorems 1.2, 1.4. Moreover, there we consider the Euler-Bernoulli operator and prove Corollary 1.5] and Theorem [1.6. In Section 6 we obtain asymptotics of the resonances and prove Theorem 1.3 .

\section{Properties of the free Resolvent}

2.1. The well-known facts. By $\mathcal{B}$ we denote the class of bounded operators. Let $\mathcal{B}_{1}$ and $\mathcal{B}_{2}$ be the trace and the Hilbert-Schmidt class equipped with the norm $\|\cdot\|_{\mathcal{B}_{1}}$ and $\|\cdot\|_{\mathcal{B}_{2}}$ correspondingly. We recall some well known facts. Let $A, B \in \mathcal{B}$ and $A B, B A, X \in \mathcal{B}_{1}$. Then

$$
\begin{gathered}
\operatorname{Tr} A B=\operatorname{Tr} B A, \\
\operatorname{det}(I+A B)=\operatorname{det}(I+B A), \\
|\operatorname{det}(I+X)| \leqslant e^{\|X\|_{\mathcal{B}_{1}},}
\end{gathered}
$$

see e.g., Sect. 3. in the book [S05]. Let the operator-valued function $X: \mathcal{D} \rightarrow \mathcal{B}_{1}$ be analytic for some domain $\mathcal{D} \subset \mathbb{C}$ and $(I+X(z))^{-1} \in \mathcal{B}$ for any $z \in \mathcal{D}$. Then for the function $F(z)=\operatorname{det}(I+X(z))$ we have

$$
F^{\prime}(z)=F(z) \operatorname{Tr}(I+X(z))^{-1} X^{\prime}(z), \quad z \in \mathcal{D} .
$$

Introduce the space $L^{p}(\mathbb{R})$ equipped by the norm $\|f\|_{p}=\left(\int_{\mathbb{R}}|f(x)|^{p} d x\right)^{\frac{1}{p}} \geqslant 0$ and we write $\|f\|=\|f\|_{2}$.

2.2. Schrödinger operator. We discuss a Schrödinger operator $h$ on $L^{2}(\mathbb{R})$ given by

$$
h=h_{0}-p, \quad h_{0}=-\frac{d^{2}}{d x^{2}}
$$

where $h_{0}$ is the unperturbed operator and the potential $p \in \mathcal{H}_{0}$.

- The operator $r_{0}(k)=\left(h_{0}-k^{2}\right)^{-1}, k \in \mathbb{C}_{+}$, is an integral operator having the kernel $r_{0}\left(x-x^{\prime}, k\right), x, x^{\prime} \in \mathbb{R}$ given by

$$
r_{0}(x, k)=\frac{1}{2 \pi} \int_{\mathbb{R}} \frac{e^{i t x}}{t^{2}-k^{2}} d t=\frac{i e^{i k|x|}}{2 k} .
$$

Define an operator-valued function $g(k)=\vartheta r_{0}(k) \varphi, k \in \mathbb{C}_{+}$, where $\vartheta^{2}, \varphi^{2} \in \mathcal{H}_{0}$. For each $k \in \mathbb{C}_{+}$the operator $g(k) \in \mathcal{B}_{j}, j=1,2$ and the mappings

$$
g: \mathbb{C}_{+} \rightarrow \mathcal{B}_{j}
$$


is analytic and it has an analytic extension into the whole complex plane without zero. Thus the operator-valued function $g: \mathbb{C} \backslash\{0\} \rightarrow \mathcal{B}_{j}$ is analytic. Moreover, we have the following estimate

$$
\|g(k)\|_{\mathcal{B}_{2}} \leqslant \frac{\|\vartheta\|\|\varphi\|}{2|k|}, \quad k \in \overline{\mathbb{C}}_{+} \backslash\{0\}
$$

Define the Fourier transformation $\Phi: L^{2}(\mathbb{R}) \rightarrow L^{2}(\mathbb{R})$ by

$$
\Phi f(\xi)=\frac{1}{\sqrt{2 \pi}} \int_{\mathbb{R}} f(x) e^{-i \xi x} d x, \quad \xi \in \mathbb{R} .
$$

Then $r_{0}(k)=\Phi^{*} \eta_{-k} \eta_{k} \Phi$, where $\eta_{k}(\xi)$ is the multiplication by $(\xi-k)^{-1}$ and we have

$$
\begin{aligned}
\|g(k)\|_{\mathcal{B}_{1}} & =\left\|\vartheta\left(h_{0}-k^{2}\right)^{-1} \varphi\right\|_{\mathcal{B}_{1}} \leqslant\left\|\vartheta \Phi^{*} \eta_{-k}\right\|_{\mathcal{B}_{2}}\left\|\eta_{k} \Phi \varphi\right\|_{\mathcal{B}_{2}} \\
& \leqslant \frac{\|\vartheta\|\|\varphi\|}{2 \pi}\left\|\eta_{-k}\right\|\left\|\eta_{k}\right\|=\frac{\|\vartheta\|\|\varphi\|}{2 \operatorname{Im} k}, \quad \operatorname{Im} k>0,
\end{aligned}
$$

since $\int_{\mathbb{R}}|\xi \pm k|^{-2} d \xi=\frac{\pi}{\operatorname{Im} k}$.

- The Schrödinger equation $-y^{\prime \prime}-p y=k^{2} y, k \in \mathbb{C} \backslash\{0\}$, has unique Jost solutions $f_{ \pm}(x, k)$ satisfying the conditions $f_{+}(x, k)=e^{i k x}, x>\gamma$ and $f_{-}(x, k)=e^{-i k x}, x<0$. For each $x \in \mathbb{R}$ the function $f_{ \pm}(x, k)$ is entire. The following identity holds true:

$$
f_{+}(x, k)=b(k) f_{-}(x, k)+a(k) f_{-}(x,-k), \quad k \in \mathbb{C} \backslash\{0\},
$$

where the functions $a, b$ are defined by

$$
a(k)=\frac{\left\{f_{-}(x, k), f_{+}(x, k)\right\}}{2 i k}, \quad b(k)=\frac{\left\{f_{+}(x, k), f_{-}(x,-k)\right\}}{2 i k},
$$

and $\{f, g\}=f g^{\prime}-f^{\prime} g$ denotes the Wronskian. The scattering matrix for the pair $h, h_{0}$ has the following form

$$
s(k)=\left(\begin{array}{cc}
a(k)^{-1} & r_{-}(k) \\
r_{+}(k) & a(k)^{-1}
\end{array}\right), \quad r_{ \pm}(k)= \pm \frac{b(\mp k)}{a(k)},
$$

where $a^{-1}$ is the transmission coefficient and $r_{ \pm}$are the reflection coefficients. It is well known the following identity $a(k)=d(k)$ for all $k \in \mathbb{C}$, where $d(k)$ is the determinant defined by (1.2). Moreover, the scattering matrix satisfies

$$
\operatorname{det} s(k)=\frac{d(-k)}{d(k)} \quad \forall \quad k>0 .
$$

- The function $d(k)$ satisfies

$$
d(k)=1+O\left(k^{-1}\right) \quad \text { as } \quad|k| \rightarrow \infty, \quad k \in \mathbb{C}_{+},
$$

uniformly on $\arg k \in[0, \pi]$. Then we can define the function $\log d(k)$ by the condition $\log d(i t)=o(1)$ as $t \rightarrow \infty$, which satisfies

$$
i \log d(k)=\frac{1}{2 k}\left(\int_{\mathbb{R}} p(x) d x+o(1)\right) \quad \text { as } \quad \operatorname{Im} k \rightarrow+\infty .
$$


2.3. The free resolvent. We rewrite the free resolvent $R_{0}(k)=\left(H_{0}-k^{4}\right)^{-1}$ in terms of the resolvent $r_{0}(k)=\left(h_{0}-k^{2}\right)^{-1}$ by

$$
R_{0}(k)=\frac{r_{0}(k)-r_{0}(i k)}{2 k^{2}}, \quad \partial R_{0}(k) \partial=-\frac{r_{0}(k)+r_{0}(i k)}{2}, \quad \forall k \in \mathbb{K}_{1} .
$$

Then the kernel of the free resolvent $R_{0}(k)$ has the form $R_{0}\left(x-x^{\prime}, k\right), x, x^{\prime} \in \mathbb{R}$, where

$$
R_{0}(x, k)=\frac{i e^{i k|x|}-e^{-k|x|}}{4 k^{3}}
$$

and satisfies

$$
R_{0}(x, k)=\frac{i-1}{4 k^{3}}-\frac{(1+i) x^{2}}{4 k}+O(1) \quad \text { as } \quad|k| \rightarrow 0
$$

locally uniformly in $x \in \mathbb{R}$. Each function $R_{0}(x, k)-\frac{i-1}{4 k^{3}}+\frac{(1+i) x^{2}}{4 k}, x \in \mathbb{R}$, is entire in $k$.

Define the operator-valued function $G(k)=\vartheta \partial R_{0}(k) \varphi, k \in \mathbb{K}_{1}$, where $\vartheta^{2}, \varphi^{2} \in \mathcal{H}_{0}$. The identity (2.11) yields that for each $k \in \mathbb{K}_{1}$ the operator $G(k) \in \mathcal{B}_{j}, j=1,2$ and the mappings

$$
G(k): \mathbb{K}_{1} \rightarrow \mathcal{B}_{j}
$$

are analytic and they have analytic extensions into whole complex plane without zero. Moreover, from (2.12) we have the following estimate

$$
\|G(k)\|_{\mathcal{B}_{2}} \leqslant \frac{\|\vartheta\|\|\varphi\|}{|k|^{2}}, \quad k \in \overline{\mathbb{K}}_{1} \backslash\{0\} .
$$

Moreover, we obtain $\partial R_{0}(k)=\Phi^{*} \rho_{i k} \rho_{k} s \Phi$, where $\rho_{k}(\xi)$ is the multiplication by $\left(\xi^{2}-k^{2}\right)^{-1}|\xi|^{\frac{1}{2}}$, $s$ is the multiplication by $\operatorname{sign} \xi$ and we have

$$
\|G(k)\|_{\mathcal{B}_{1}} \leqslant\left\|\vartheta \Phi^{*} \rho_{i k}\right\|_{\mathcal{B}_{2}}\left\|\rho_{k} \Phi \varphi\right\|_{\mathcal{B}_{2}} \leqslant \frac{\|\vartheta\|\|\varphi\|}{2 \pi}\left\|\rho_{i k}\right\|\left\|\rho_{k}\right\|=\frac{\|\vartheta\|\|\varphi\|}{4 \operatorname{Re} k \operatorname{Im} k}
$$

$k \in \mathbb{K}_{1}$, since $\int_{\mathbb{R}}|\xi|\left|\xi^{2} \pm k^{2}\right|^{-2} d \xi=\frac{\pi}{4 \operatorname{Re} k \operatorname{Im} k}$.

2.4. Resolvent estimates. The operator $H_{0}$ is self-adjoint on the form domain given by $\mathscr{D}\left(H_{0}\right)=\left\{y, y^{\prime \prime} \in L^{2}(\mathbb{R})\right\}$. The quadratic form $(V y, y)$ is defined by $(V y, y)=-\left(p y^{\prime}, y^{\prime}\right)+$ $(q y, y), y \in \mathscr{D}\left(H_{0}\right)$, where $(u, v)$ is the scalar product in $L^{2}(\mathbb{R})$. Then the standard arguments (see e.g., [K03]) give

$$
|(V y, y)| \leqslant \frac{1}{2}\left\|y^{\prime \prime}\right\|^{2}+C\|y\|^{2} \quad \forall \quad y \in \mathscr{D}\left(H_{0}\right)
$$

for some constant $C>0$. Then the KLMN Theorem (see [RS75, Th X.17]) yields that there exists a unique self-adjoint operator $H=H_{0}+V$ with the form domain $\mathscr{D}(H)=\mathscr{D}\left(H_{0}\right)$ and

$$
\left(H y, y_{1}\right)=\left(H_{0} y, y_{1}\right)+\left(V y, y_{1}\right) \quad \forall \quad y, y_{1} \in \mathscr{D}\left(H_{0}\right)
$$

In order to study the determinant we need to consider $Y_{0}$. The definitions (1.3), (1.4) imply

$$
Y_{0}=\left(\begin{array}{cc}
(2 p)^{\frac{1}{2}} \partial R_{0} \partial|2 p|^{\frac{1}{2}} & (2 p)^{\frac{1}{2}} \partial R_{0}|q|^{\frac{1}{2}} \\
q^{\frac{1}{2}} R_{0} \partial|2 p|^{\frac{1}{2}} & q^{\frac{1}{2}} R_{0}|q|^{\frac{1}{2}}
\end{array}\right) .
$$

We introduce the operator-valued function $Y$ by

$$
Y(k)=V_{2} R(k) V_{1}, \quad k \in \mathbb{K}_{1} .
$$

This function satisfies the standard identity

$$
(I-Y(k))\left(I+Y_{0}(k)\right)=I \quad \forall k \in \mathbb{K}_{1} \backslash \sigma_{d},
$$


where $\sigma_{d}$ is the set of the zeros of the function $D$ in $\overline{\mathbb{K}}_{1}$.

Lemma 2.1. Let $p, q \in \mathcal{H}_{0}$. Then

i) The operator $Y_{0}(k) \in \mathcal{B}_{j}, j=1,2$ for each $k \in \mathbb{K}_{1}$, the operator-valued function $Y_{0}: \mathbb{K}_{1} \rightarrow$ $\mathcal{B}_{j}$ is analytic and has an analytic extension into the whole complex plane without zero. The operator-valued function $k^{3} Y_{0}(k)$ is entire. Moreover, $Y_{0}$ satisfies

$$
\begin{gathered}
\left\|Y_{0}(k)\right\|_{\mathcal{B}_{2}} \leqslant \frac{C}{|k|} \\
\left\|Y_{0}(k)\right\|_{\mathcal{B}_{1}} \leqslant(\|2 p\|+\|q\|)\left(\frac{1}{\operatorname{Re} k}+\frac{1}{\operatorname{Im} k}\right)\left(1+\frac{1}{|k|}\right)^{2},
\end{gathered}
$$

$k \in \mathbb{K}_{1}$, for some constant $C=C(p, q)>0$.

ii) The operator $Y(k) \in \mathcal{B}_{1}$ for each $k \in \mathbb{K}_{1} \backslash \sigma_{d}$ and the operator-valued function $Y$ : $\mathbb{K}_{1} \backslash \sigma_{d} \rightarrow \mathcal{B}_{1}$ is analytic and has a meromorphic extension from $\mathbb{K}_{1} \backslash \sigma_{d}$ into the whole complex plane. Moreover, $Y$ satisfies

$$
\begin{gathered}
\|Y(k)\|_{\mathcal{B}_{2}}=\frac{O(1)}{|k|}, \\
\left\|Y(k)-Y_{0}(k)\right\|_{\mathcal{B}_{2}}=\frac{O(1)}{|k|^{2}},
\end{gathered}
$$

as $k \in \overline{\mathbb{K}}_{1},|k| \rightarrow \infty$ and uniformly in $\arg k \in[0, \pi]$.

Proof. i) Substituting the identity (2.12) into the definition (1.4) we obtain (2.21). Substituting the identities (2.11) into (2.18) and using the facts about the mappings $g, G$ in (2.6), (2.14) we deduce that the operator-valued function $Y_{0}: \mathbb{K}_{1} \rightarrow \mathcal{B}_{1}$ is analytic and has an analytic extension into the whole complex plane without zero. The asymptotics (2.13) shows that the operator-valued function $k^{3} Y_{0}(k)$ is entire.

Using the estimates (2.9) we obtain for $\operatorname{Im} k>0$ :

$$
\begin{array}{r}
\left\|p^{\frac{1}{2}} r_{0}(k)|p|^{\frac{1}{2}}\right\|_{\mathcal{B}_{1}} \leqslant \frac{\|p\|_{1}}{2 \operatorname{Im} k}, \quad\left\|q^{\frac{1}{2}} r_{0}(k)|q|^{\frac{1}{2}}\right\|_{\mathcal{B}_{1}} \leqslant \frac{\|q\|_{1}}{2 \operatorname{Im} k}, \\
\left\|p^{\frac{1}{2}} r_{0}(k)|q|^{\frac{1}{2}}\right\|_{\mathcal{B}_{1}} \leqslant \frac{\left(\|p\|_{1}\|q\|_{1}\right)^{\frac{1}{2}}}{2 \operatorname{Im} k},
\end{array}
$$

and the similar estimates with $r_{0}(i k)$. These estimates and the relations (2.15), (2.18) give

$$
\left\|Y_{0}(k)\right\|_{\mathcal{B}_{1}} \leqslant \frac{1}{4}\left(\|2 p\|_{1}^{\frac{1}{2}}+\|q\|_{1}^{\frac{1}{2}}\right)^{2}\left(\frac{1}{\operatorname{Re} k}+\frac{1}{\operatorname{Im} k}\right)\left(1+\frac{1}{|k|}\right)^{2},
$$

which yields (2.22).

ii) For $k \in \mathbb{K}_{1} \backslash \sigma_{d}$ identity (2.20) gives

$$
Y(k)=I-\left(I+Y_{0}(k)\right)^{-1}=Y_{0}(k)\left(I+Y_{0}(k)\right)^{-1} \in \mathcal{B}_{j}, \quad j=1,2,
$$

and, since $Y_{0}(k)$ is analytic in $\mathbb{K}_{1}, Y(k)$ is analytic in $\mathbb{K}_{1} \backslash \sigma_{d}$. Due to the analytic Fredholm theorem, see [RS72, Th VI.14], the function $Y(k)$ has a meromorphic extension into the whole complex plane. The estimate (2.21) implies the asymptotics (2.23). Moreover,

$$
Y(k)-Y_{0}(k)=Y_{0}(k)\left(\left(I+Y_{0}(k)\right)^{-1}-I\right),
$$

which yields (2.24). 


\section{The SCATTERING MATRIX.}

3.1. The spectral representation for $H_{0}$. Define a unitary operator

$$
\mathcal{F}: L^{2}(\mathbb{R}) \rightarrow L^{2}\left(\mathbb{R}_{+}, d k\right) \oplus L^{2}\left(\mathbb{R}_{+}, d k\right)
$$

by

$$
\mathcal{F} f(k)=\left(\begin{array}{c}
\widehat{f}(k) \\
\widehat{f}(-k)
\end{array}\right), \quad k \in \mathbb{R}_{+}, \quad \widehat{f}(k)=\Phi f(k)=\frac{1}{\sqrt{2 \pi}} \int_{\mathbb{R}} f(x) e^{-i k x} d x .
$$

The identity $\left(\Phi H_{0} \Phi^{*} \widehat{f}\right)(k)=k^{4} \widehat{f}(k), k \in \mathbb{R}$, implies that $\mathcal{F} H_{0} \mathcal{F}^{*}$ is the operator of multiplication by $k^{4}$ in $L^{2}\left(\mathbb{R}_{+}, d k\right) \oplus L^{2}\left(\mathbb{R}_{+}, d k\right)$.

Introduce the operators $\psi_{1}(k): L^{2}(\mathbb{R}) \oplus L^{2}(\mathbb{R}) \rightarrow \mathbb{C}^{2}$ and $\psi_{2}(k): \mathbb{C}^{2} \rightarrow L^{2}(\mathbb{R}) \oplus L^{2}(\mathbb{R})$ for each $k>0$ by

$$
\begin{array}{r}
\psi_{1}(k) f=\left(\begin{array}{l}
\psi_{11}(k) \\
\psi_{21}(k)
\end{array}\right) f=\mathcal{F} V_{1} f(k)=\left(\begin{array}{c}
\Phi V_{1} f(k) \\
\Phi V_{1} f(-k)
\end{array}\right)=\int_{\mathbb{R}} \psi_{1}(x, k) f(x) d x, \\
\left(\psi_{2}(k) c\right)(x)=\left(\psi_{12}(k), \psi_{22}(k)\right) c=\frac{1}{\sqrt{2 \pi}} V_{2}\left(e^{i k x}, e^{-i k x}\right) c=\psi_{2}(x, k) c,
\end{array}
$$

where $f \in L^{2}(\mathbb{R}) \oplus L^{2}(\mathbb{R})$ and $c \in \mathbb{C}^{2}$. Here $\psi_{j 1}(k): L^{2}(\mathbb{R}) \oplus L^{2}(\mathbb{R}) \rightarrow \mathbb{C}$ and $\psi_{j 2}(k): \mathbb{C} \rightarrow$ $L^{2}(\mathbb{R}) \oplus L^{2}(\mathbb{R}), j=1,2$, and the kernels $\psi_{j}(\cdot, \cdot), j=1,2$ have the forms:

$$
\begin{array}{r}
\psi_{1}(x, k)=\frac{1}{\sqrt{2 \pi}}\left(\begin{array}{cc}
i k|2 p(x)|^{\frac{1}{2}} e^{-i k x} & |q(x)|^{\frac{1}{2}} e^{-i k x} \\
-i k|2 p(x)|^{\frac{1}{2}} e^{i k x} & |q(x)|^{\frac{1}{2}} e^{i k x}
\end{array}\right), \\
\psi_{2}(x, k)=\frac{1}{\sqrt{2 \pi}}\left(\begin{array}{cc}
i k(2 p(x))^{\frac{1}{2}} e^{i k x} & -i k(2 p(x))^{\frac{1}{2}} e^{-i k x} \\
q^{\frac{1}{2}}(x) e^{i k x} & q^{\frac{1}{2}}(x) e^{-i k x}
\end{array}\right) .
\end{array}
$$

It is clear that the operator-valued functions $\psi_{j}(k), k \in \mathbb{R}_{+}, j=1,2$ have analytic extensions from $\mathbb{R}_{+}$into the whole complex plane. Then we can introduce the operators $\Psi_{1}(k)$ and $\Psi_{2}(k)$ by

$$
\Psi_{1}(k)=\left(\begin{array}{c}
\psi_{1}(i k) \\
\psi_{1}(k)
\end{array}\right), \quad \Psi_{2}(k)=\left(i \psi_{2}(i k), \psi_{2}(k)\right), \quad k \in \mathbb{C} .
$$

Lemma 3.1. Let $p, q \in \mathcal{H}_{0}$. Then the operator-valued functions $\psi_{j}(k), j=1,2$, are entire and satisfy

$$
\left\|\psi_{j j}(k)\right\| \leqslant C(k) e^{\gamma(\operatorname{Im} k)_{+}}, \quad\left\|\psi_{j \ell}(k)\right\| \leqslant C(k) e^{\gamma(\operatorname{Im} k)_{-}}
$$

for all $k \in \mathbb{C}, j, \ell=1,2, j \neq \ell$, where

$$
\left(a_{ \pm}\right)=\max \{ \pm a, 0\}, \quad a \in \mathbb{R}, \quad C(k)=\frac{1}{\sqrt{2 \pi}}\left(2|k|^{2}\|p\|_{1}+\|q\|_{1}\right)^{\frac{1}{2}} .
$$

Proof. The operator $\psi_{1}(k), k \in \mathbb{R}_{+}$has the kernel $\psi_{1}(x, k)$ given by (3.3) . Let $g=$ $\psi_{1}(k) f, f=\left(f_{1}, f_{2}\right) \in L^{2}(\mathbb{R}) \oplus L^{2}(\mathbb{R})$. We have

$$
g=\left(\begin{array}{c}
g_{+} \\
g_{-}
\end{array}\right)=\int_{0}^{\gamma} \psi_{1}(x, k) f(x) d x=\frac{1}{\sqrt{2 \pi}} \int_{0}^{\gamma}\left(\begin{array}{c}
e^{-i k x}\left(i k|2 p(x)|^{\frac{1}{2}} f_{1}(x)+|q(x)|^{\frac{1}{2}} f_{2}(x)\right) \\
e^{i k x}\left(-i k|2 p(x)|^{\frac{1}{2}} f_{1}(x)+|q(x)|^{\frac{1}{2}} f_{2}(x)\right)
\end{array}\right) d x,
$$

and

$$
\left|g_{ \pm}\right|^{2} \leqslant e^{2 \gamma(\operatorname{Im} k)_{ \pm}} \frac{1}{2 \pi}\|f\|^{2} \int_{0}^{\gamma}\left(|k|^{2}|2 p(x)|+|q(x)|\right) d x .
$$

This yields the estimates (3.5) for $\psi_{1}(k)$. The proof for $\psi_{2}(k)$ is similar. 
Introduce finite rank operators $P_{1}(k), P_{2}(k), k \in \mathbb{C} \backslash\{0\}$, acting on $L^{2}(\mathbb{R}) \oplus L^{2}(\mathbb{R})$, by

$$
\begin{gathered}
P_{1}(k)=c_{k} \psi_{2}(k) \psi_{1}(k), \quad c_{k}=\frac{\pi}{i 2 k^{3}}, \\
P_{2}(k)=P_{1}(i k)+P_{1}(k)=c_{k} \Psi_{2}(k) \Psi_{1}(k) .
\end{gathered}
$$

The operators $P_{1}(k), P_{2}(k)$ are analytic in the domain $\mathbb{C} \backslash\{0\}$. Below we need the following simple identities.

Lemma 3.2. Let $p, q \in \mathcal{H}_{0}$. Then for any $k \in \mathbb{C} \backslash\{0\}$ the operators $P_{1}(k), P_{2}(k)$ satisfy

$$
\begin{gathered}
P_{1}(k)=Y_{0}(i k)-Y_{0}(k), \\
P_{2}(k)=Y_{0}(-k)-Y_{0}(k) .
\end{gathered}
$$

Proof. The identities (2.5), (2.11) yield

$$
R_{0}(y, k)-R_{0}(y, i k)=\frac{r_{0}(y, k)-r_{0}(y,-k)}{2 k^{2}}=\frac{i \cos k y}{2 k^{3}}, \quad \forall \quad(k, y) \in \mathbb{C} \times \mathbb{R}, \quad k \neq 0 .
$$

For all $f \in L^{2}(\mathbb{R}) \oplus L^{2}(\mathbb{R})$ the definitions (3.2) imply

$$
\psi_{2}(k) \psi_{1}(k) f=\frac{1}{2 \pi} V_{2}\left(e^{i k x}, e^{-i k x}\right) \int_{\mathbb{R}}\left(\begin{array}{c}
e^{-i k x^{\prime}} \\
e^{i k x^{\prime}}
\end{array}\right) V_{1} f\left(x^{\prime}\right) d x^{\prime}=\frac{1}{\pi} V_{2} \int_{\mathbb{R}} \cos k\left(x-x^{\prime}\right) V_{1} f\left(x^{\prime}\right) d x^{\prime} .
$$

This identity together with the identity (3.9) and the definition (1.4) gives

$$
Y_{0}(i k)-Y_{0}(k)=V_{2}\left(R_{0}(i k)-R_{0}(k)\right) V_{1}=c_{k} \psi_{2}(k) \psi_{1}(k),
$$

which yields the identity (3.7).

The identities

$$
Y_{0}(-k)-Y_{0}(k)=Y_{0}(-k)-Y_{0}(i k)+Y_{0}(i k)-Y_{0}(k)=P_{1}(i k)+P_{1}(k)
$$

give (3.8).

3.2. The scattering matrix. We define the S-matrix for the operators $H_{0}, H$. It is well known that the wave operators $W_{ \pm}=W_{ \pm}\left(H, H_{0}\right)$ for the pair $H_{0}, H$, given by

$$
W_{ \pm}=s-\lim e^{i t H} e^{-i t H_{0}} \quad \text { as } \quad t \rightarrow \pm \infty,
$$

exist and are complete, i.e., $\operatorname{Ran} W_{ \pm}=\mathscr{H}_{a c}(H)$. The scattering operator $\mathcal{S}=W_{+}^{*} W_{-}$is unitary. The operators $H_{0}$ and $\mathcal{S}$ commute and thus are simultaneously diagonalizable:

$$
L^{2}(\mathbb{R})=\int_{\mathbb{R}_{+}}^{\oplus} \mathscr{H}_{\lambda} d \lambda, \quad H_{0}=\int_{\mathbb{R}_{+}}^{\oplus} \lambda I_{\lambda} d \lambda, \quad \mathcal{S}=\int_{\mathbb{R}_{+}}^{\oplus} S\left(\lambda^{\frac{1}{4}}\right) d \lambda ;
$$

here $I_{\lambda}$ is the identity in the fiber space $\mathscr{H}_{\lambda}=\mathbb{C}^{2}$ and $S(k), k=\lambda^{\frac{1}{4}}>0$ is the scattering matrix (which is a $2 \times 2$ matrix-valued function of $k>0$ in our case) for the pair $H_{0}, H$.

The operator $\mathcal{S}=W_{+}^{*} W_{-}$commutes with the operator $H_{0}$ and the operator $\mathcal{F} H_{0} \mathcal{F}^{*}$ is the operator of multiplication by $k^{4}$ in $L^{2}\left(\mathbb{R}_{+}, d k\right) \oplus L^{2}\left(\mathbb{R}_{+}, d k\right)$. Then the operator $\mathcal{F} \mathcal{S} \mathcal{F}^{*}$ acts in the space $L^{2}\left(\mathbb{R}_{+}, d k\right) \oplus L^{2}\left(\mathbb{R}_{+}, d k\right)$ as multiplication by a $2 \times 2$ matrix-valued function $S(k)$.

The scattering matrix $S(k)$ is a continuous $2 \times 2$ matrix-valued function in $\mathbb{R}_{+} \backslash \sigma_{d}$, where $\sigma_{d}$ is the set of the zeros of the function $D$ in $\overline{\mathbb{K}}_{1}$, and satisfies

$$
S(k)=\left(\begin{array}{ll}
s_{11}(k) & s_{12}(k) \\
s_{21}(k) & s_{22}(k)
\end{array}\right)=\mathbb{1}_{2}+c_{k} \mathcal{A}(k), \quad \forall k=\lambda^{\frac{1}{4}}>0, \quad c_{k}=\frac{\pi}{i 2 k^{3}}
$$


(see, e.g., [RS79]), where $\mathbb{1}_{N}$ is the $N \times N$ identity matrix and $\mathcal{A}(k)$ is the scattering amplitude given by

$$
\mathcal{A}(k)=\mathcal{A}_{0}(k)-\mathcal{A}_{1}(k)
$$

where the Born term $\mathcal{A}_{0}(k)$ and the term $\mathcal{A}_{1}(k)$ have the form

$$
\mathcal{A}_{0}(k)=\psi_{1}(k) \psi_{2}(k), \quad \mathcal{A}_{1}(k)=\psi_{1}(k) Y(k) \psi_{2}(k) .
$$

The operator-valued functions $\psi_{1}(k), \psi_{2}(k)$ are analytic in $\mathbb{C}$, then the matrix-valued function $\mathcal{A}_{0}(k)$ has an analytic extension from $\mathbb{R}_{+}$into the whole complex plane.

3.3. The scattering amplitude. Now we consider the scattering amplitude $\mathcal{A}$.

Lemma 3.3. i) Let $p, q \in \mathcal{H}_{0}$. Then the scattering amplitude $\mathcal{A}(k), k \in \mathbb{R}_{+}$has a meromorphic extension from $\mathbb{R}_{+}$into the whole complex plane. Moreover, the matrix-valued function $\mathcal{A}_{0}(k)$ is entire and satisfies

$$
\mathcal{A}_{0}(k)=\frac{1}{2 \pi}\left(\begin{array}{cc}
\alpha_{1}(k) & \alpha_{2}(k) \\
\alpha_{2}(-k) & \alpha_{1}(k)
\end{array}\right)
$$

for all $k \in \mathbb{K}_{1}$, where

$$
\begin{gathered}
\alpha_{1}(k)=q_{0}-2 k^{2} p_{0}, \quad \alpha_{2}(k)=\sqrt{2 \pi}\left(2 k^{2} \widehat{p}(2 k)+\widehat{q}(2 k)\right), \\
p_{0}=\int_{\mathbb{R}} p(x) d x, \quad q_{0}=\int_{\mathbb{R}} q(x) d x, \quad \widehat{f}(k)=\frac{1}{\sqrt{2 \pi}} \int_{\mathbb{R}} f(x) e^{-i k x} d x,
\end{gathered}
$$

the matrix-valued functions $\mathcal{A}_{0}(k), \mathcal{A}_{1}(k)$ satisfy

$$
\begin{gathered}
\mathcal{A}_{0}(k)=\left(\begin{array}{cc}
O\left(k^{2}\right) & e^{2 \gamma \operatorname{Im} k} o\left(k^{2}\right) \\
o\left(k^{2}\right) & O\left(k^{2}\right)
\end{array}\right), \quad \mathcal{A}_{0}(i k)=\left(\begin{array}{cc}
O\left(k^{2}\right) & e^{2 \gamma \operatorname{Re} k} o\left(k^{2}\right) \\
o\left(k^{2}\right) & O\left(k^{2}\right)
\end{array}\right), \\
\mathcal{A}_{1}(k)=\left(\begin{array}{cc}
e^{\gamma \operatorname{Im} k} O(k) & e^{2 \gamma \operatorname{Im} k} O(k) \\
O(k) & e^{\gamma \operatorname{Im} k} O(k)
\end{array}\right),
\end{gathered}
$$

as $|k| \rightarrow \infty, k \in \overline{\mathbb{K}}_{1}$ uniformly in $\arg k \in\left[0, \frac{\pi}{2}\right]$.

ii) Let $(p, q) \in \mathcal{H}_{1} \times \mathcal{H}_{0}$. Then the functions $\mathcal{A}_{0}(k), \mathcal{A}_{1}(k)$ satisfy

$$
\begin{gathered}
\mathcal{A}_{0}(k)=\frac{k}{2 \pi}\left(\begin{array}{cc}
-2 k\left(p_{0}+O\left(k^{-2}\right)\right) & i e^{-i 2 k \gamma}\left(p_{+}+o(1)+e^{-2 \gamma \operatorname{Im} k} O(1)\right) \\
i p_{-}+o(1)+e^{-2 \gamma \operatorname{Im} k} O(1) & -2 k\left(p_{0}+O\left(k^{-2}\right)\right)
\end{array}\right), \\
\mathcal{A}_{0}(i k)=-\frac{k}{2 \pi}\left(\begin{array}{cc}
2 k\left(p_{0}+O\left(k^{-2}\right)\right) & e^{2 k \gamma}\left(p_{+}+o(1)+e^{-2 \gamma \operatorname{Re} k} O(1)\right) \\
p_{-}+o(1)+e^{-2 \gamma \operatorname{Re} k} O(1) & 2 k\left(p_{0}+O\left(k^{-2}\right)\right)
\end{array}\right), \\
\mathcal{A}_{1}(k)=\left(\begin{array}{cc}
e^{\gamma \operatorname{Im} k} O(1) & e^{2 \gamma \operatorname{Im} k} O(1) \\
O(1) & e^{\gamma \operatorname{Im} k} O(1)
\end{array}\right),
\end{gathered}
$$

as $|k| \rightarrow \infty, k \in \overline{\mathbb{K}}_{1}$, uniformly in $\arg k \in\left[0, \frac{\pi}{2}\right]$, where $p_{+}=p(\gamma-0), p_{-}=p(+0)$.

Proof. i) The operator-valued functions $\psi_{1}, \psi_{2}$ are entire, then the function $\mathcal{A}_{0}(k)$ has an analytic extension from $\mathbb{R}_{+}$into the whole complex plane. Due to Lemma $\left.2.1 \mathrm{ii}\right)$, the function $\mathcal{A}_{1}(k)$ has a meromorphic extension from $\mathbb{R}_{+}$onto the whole complex plane. Then the scattering amplitude $\mathcal{A}(k)$ has a meromorphic extension from $\mathbb{R}_{+}$into the whole complex plane.

The definitions (3.2) and (3.13) give

$$
\mathcal{A}_{0}(k)=\int_{\mathbb{R}} \psi_{1}(x, k) \psi_{2}(x, k) d x,
$$




$$
\mathcal{A}_{1}(k)=\left(\begin{array}{l}
\psi_{11}(k) \\
\psi_{21}(k)
\end{array}\right) Y(k)\left(\psi_{12}(k), \psi_{22}(k)\right) .
$$

Substituting the identities (3.3) into (3.21) we obtain the identity (3.14), which yields the asymptotics (3.16). The estimates (2.23) and (3.5) and the identity (3.22) give the asymptotics (3.17).

ii) Let $|k| \rightarrow \infty, k \in \overline{\mathbb{K}}_{1}$. The integration by parts gives

$$
\begin{gathered}
\alpha_{2}(k)=\int_{0}^{\gamma}\left(2 k^{2} p(x)+q(x)\right) e^{-2 i k x} d x=i k\left(p_{+}+o(1)\right) e^{-i 2 k \gamma}+O(k), \\
\alpha_{2}(-k)=\int_{0}^{\gamma}\left(2 k^{2} p(x)+q(x)\right) e^{2 i k x} d x=i k\left(p_{-}+o(1)\right)+e^{i 2 k \gamma} O(k),
\end{gathered}
$$

Substituting these asymptotics and the definition (3.15) into the identity (3.14) we obtain (3.18). Similarly,

$$
\begin{array}{r}
\alpha_{2}(i k)=\int_{0}^{\gamma}\left(-2 k^{2} p(x)+q(x)\right) e^{2 k x} d x=-k\left(p_{+}+o(1)\right) e^{2 k \gamma}+O(k), \\
\alpha_{2}(-i k)=\int_{0}^{\gamma}\left(-2 k^{2} p(x)+q(x)\right) e^{-2 k x} d x=-k\left(p_{-}+o(1)\right)+e^{-2 k \gamma} O(k),
\end{array}
$$

which yields (3.19).

The definition (3.13) and the estimates (2.24) and (3.5) give

$$
\mathcal{A}_{1}(k)=\psi_{1}(k) Y_{0}(k) \psi_{2}(k)+\left(\begin{array}{cc}
e^{\gamma \operatorname{Im} k} O(1) & e^{2 \gamma \operatorname{Im} k} O(1) \\
O(1) & e^{\gamma \operatorname{Im} k} O(1)
\end{array}\right) .
$$

The identities (2.18) and (3.3) imply

$$
\begin{aligned}
& \psi_{11}(k) Y_{0}(k) \psi_{12}(k)=\frac{1}{2 \pi} \iint_{[0, \gamma]^{2}} e^{-i k\left(x-x^{\prime}\right)}\left(-4 k^{2} p(x) p\left(x^{\prime}\right) \frac{\partial^{2} R_{0}\left(x, x^{\prime}, k\right)}{\partial x^{2}}\right. \\
& \left.+2 i k\left(p(x) q\left(x^{\prime}\right)+p\left(x^{\prime}\right) q(x)\right) \frac{\partial R_{0}\left(x, x^{\prime}, k\right)}{\partial x}+q(x) q\left(x^{\prime}\right) R_{0}\left(x, x^{\prime}, k\right)\right) d x d x^{\prime} .
\end{aligned}
$$

Substituting the kernel (2.12) into the last identity and integrating by parts in the first term we obtain

$$
\psi_{11}(k) Y_{0}(k) \psi_{12}(k)=e^{\gamma \operatorname{Im} k} O(1) .
$$

Similarly,

$$
\begin{gathered}
\psi_{11}(k) Y_{0}(k) \psi_{22}(k)=e^{2 \gamma \operatorname{Im} k} O(1), \quad \psi_{21}(k) Y_{0}(k) \psi_{12}(k)=O(1), \\
\psi_{21}(k) Y_{0}(k) \psi_{22}(k)=e^{\gamma \operatorname{Im} k} O(1),
\end{gathered}
$$

which yields

$$
\psi_{1}(k) Y_{0}(k) \psi_{2}(k)=\left(\begin{array}{cc}
e^{\gamma \operatorname{Im} k} O(1) & e^{2 \gamma \operatorname{Im} k} O(1) \\
O(1) & e^{\gamma \operatorname{Im} k} O(1)
\end{array}\right) .
$$

Substituting this asymptotics into (3.23) we obtain the asymptotics (3.20). 


\section{The DETERMinAnT}

4.1. Asymptotics of the determinant. Lemma $2.1 \mathrm{i})$ shows that $Y_{0}(k) \in \mathcal{B}_{1}$, then the determinant $D(k)=\operatorname{det}\left(I+Y_{0}(k)\right), k \in \mathbb{K}_{1}$ is well defined.

Lemma 4.1. Let $p, q \in \mathcal{H}_{0}$. Then

i) The determinant $D(k)=\operatorname{det}\left(I+Y_{0}(k)\right)$ is analytic in $\mathbb{K}_{1}$ and has an analytic extension from $\mathbb{K}_{1}$ into the whole complex plane without zero, such that the function $k^{4} D(k)$ is entire.

ii) The function $D(k)$ is real on the line $e^{i \frac{\pi}{4}} \mathbb{R}$.

Proof. i) Due to Lemma 2.1 i) the operator-valued function $Y_{0}(k)$, and then the determinant $D(k)$, is analytic in $k \in \mathbb{K}_{1}$ and has an analytic extension from $k \in \mathbb{K}_{1}$ into the whole complex plane without zero. It is proved in [BK16] that the function $k^{4} D(k)$ is entire, the proof is rather technical.

ii) The identity $(2.12)$ shows that $R_{0}(k)$ is real on the line $e^{i \frac{\pi}{4}} \mathbb{R}$, then $Y_{0}(k)$ is real also. Therefore, $D(k)$ is real on this line.

The identities (2.5), (2.11), (2.18) imply

$$
\begin{array}{r}
\operatorname{Tr} Y_{0}(k)=\int_{\mathbb{R}}\left(-\left(r_{0}(x, x, k)+r_{0}(x, x, i k)\right) p(x)+\frac{\left(r_{0}(x, x, k)-r_{0}(x, x, i k)\right) q(x)}{2 k^{2}}\right) d x \\
=-\frac{(1+i) p_{0}}{2 k}-\frac{(1-i) q_{0}}{4 k^{3}},
\end{array}
$$

where $p_{0}=\int_{\mathbb{R}} p(x) d x, q_{0}=\int_{\mathbb{R}} q(x) d x$. The estimates (2.22) give $\left\|Y_{0}(k)\right\|_{\mathcal{B}_{1}}=O\left(k^{-1}\right)$ as $k \rightarrow e^{i \frac{\pi}{4}} \infty$. We can define the branch $\log D(k)$, for $k \in \mathbb{K}_{1}$ and $|k|$ large enough, by

$$
\log D(k)=o(1) \quad \text { as } \quad k \rightarrow e^{i \frac{\pi}{4}} \infty .
$$

We need the following standard results.

Lemma 4.2. Let $p, q \in \mathcal{H}_{0}$. Then the function $\log D(k)$ satisfies

$$
\begin{gathered}
\left|\log D(k)+\sum_{n=1}^{N} \frac{1}{n} \operatorname{Tr}\left(-Y_{0}(k)\right)^{n}\right| \leqslant \frac{C_{1}}{|k|^{N+1}}, \quad \forall \quad N \geqslant 1, \\
\log D(k)=-\sum_{n=1}^{\infty} \frac{1}{n} \operatorname{Tr}\left(-Y_{0}(k)\right)^{n},
\end{gathered}
$$

for any $k \in \mathbb{K}_{1},|k|$ large enough, and for some $C_{1}>0$, where the series converges absolutely and uniformly in $k$. Furthermore, the function $\log D$ satisfies the asymptotics

$$
\log D(k)=-\frac{(1+i) p_{0}}{2 k}+\frac{O(1)}{k^{2}} \quad \text { as } \quad|k| \rightarrow \infty, \quad k \in \overline{\mathbb{K}}_{1}
$$

uniformly in $\arg k \in\left[0, \frac{\pi}{2}\right]$.

Proof. Let $k \in \mathbb{K}_{1}$. The estimate (2.21) gives

$$
\left|\operatorname{Tr}\left(Y_{0}(k)\right)^{n}\right| \leqslant\left\|Y_{0}(k)\right\|_{\mathcal{B}_{2}}^{n} \leqslant\left(\frac{C}{|k|}\right)^{n}, \quad n \geqslant 2 .
$$

Then the series (4.3) converges absolutely and uniformly and it is well-known that the sum is equal to $\log D(k)$ (see [RS78, Lm XIII.17.6]). Using the estimates (4.5) we obtain (4.2). The estimate (4.2) together with the identity (4.1) gives the asymptotics (4.4). 
4.2. Identities for the determinant and S-matrix. Asymptotics of the determinant in $\mathbb{C}_{-}$in the case of the Schrödinger operator is obtained from the asymptotics in $\mathbb{C}_{+}$and the identity (2.10). In order to determine asymptotics of the determinant in $\mathbb{K}_{2}, \mathbb{K}_{3}, \mathbb{K}_{4}$ for the case of fourth order operators we need some additional identities. The situation for third order operators is described in [K16].

Recall that the $S$-matrix $S(k)$ is a meromorphic matrix-valued function and satisfies the identity $S(k)=\mathbb{1}_{2}+c_{k}\left(\mathcal{A}_{0}(k)-\mathcal{A}_{1}(k)\right)$, see (3.11), (3.12), where $\mathcal{A}_{0}(k)=\psi_{1}(k) \psi_{2}(k)$ is the Born approximation for the scattering amplitude $\mathcal{A}=\mathcal{A}_{0}-\mathcal{A}_{1}, \mathcal{A}_{1}(k)=\psi_{1}(k) Y(k) \psi_{2}(k)$.

Introduce the $4 \times 4$ matrix-valued function $\Omega(k), k \in \mathbb{K}_{1} \backslash \sigma_{d}$ by

$$
\Omega(k)=\mathbb{1}_{4}+c_{k}\left(\Omega_{0}(k)-\Omega_{1}(k)\right), \quad c_{k}=\frac{\pi}{i 2 k^{3}},
$$

where the "Born" term $\Omega_{0}(k)$ has the form

$$
\Omega_{0}(k)=\Psi_{1}(k) \Psi_{2}(k),
$$

$\Psi_{1}, \Psi_{2}$ are given by (3.4), and

$$
\Omega_{1}(k)=\Psi_{1}(k) Y(k) \Psi_{2}(k),
$$

The function $\Omega_{0}(k)$ has an analytic extension and the functions $\Omega_{1}(k), \Omega(k)$ have meromorphic extensions from $\mathbb{K}_{1} \backslash \sigma_{d}$ onto the whole complex plane.

Lemma 4.3. Let $p, q \in \mathcal{H}_{0}$, and let $k \in \mathbb{C} \backslash\{0\}$. Then the determinant $D$ satisfies

$$
\begin{gathered}
D(i k)=D(k) \operatorname{det} S(k), \\
D(-k)=D(k) \operatorname{det} \Omega(k) .
\end{gathered}
$$

The function $\operatorname{det} S(k)$ is continuous in $\mathbb{R}_{+}$.

Proof. The identities (3.6), (3.7) and (2.20) give

$$
\begin{array}{r}
D(i k)=\operatorname{det}\left(1+Y_{0}(i k)\right)=\operatorname{det}\left(1+Y_{0}(k)+P_{1}(k)\right)=D(k) \operatorname{det}\left(1+(1-Y(k)) P_{1}(k)\right) \\
=D(k) \operatorname{det}\left(1+c_{k}(1-Y(k)) \psi_{2}(k) \psi_{1}(k)\right) .
\end{array}
$$

The definitions (3.11), (3.12) give

$$
S(k)=1+c_{k} \psi_{1}(k)(1-Y(k)) \psi_{2}(k) .
$$

The identity (2.2) implies (4.9).

Similarly, the identities (3.6), (3.8) and (2.20) give

$$
\begin{aligned}
D(-k)=\operatorname{det}\left(1+Y_{0}(-k)\right)=\operatorname{det}\left(1+Y_{0}(k)\right. & \left.+P_{2}(k)\right)=D(k) \operatorname{det}\left(1+(1-Y(k)) P_{2}(k)\right) \\
& =D(k) \operatorname{det}\left(1+c_{k}(1-Y(k)) \Psi_{2}(k) \Psi_{1}(k)\right) .
\end{aligned}
$$

Then the identity (2.2) and the definition (4.6) imply (4.10).

Due to Lemma 3.3, the function $\operatorname{det} S(k)$ is continuous in $k \in \mathbb{R}_{+} \backslash \sigma_{d}$ and it has a meromorphic extension from $\mathbb{R}_{+}$onto $\mathbb{C}$. Moreover, if $k \in \sigma_{d} \cap \mathbb{R}_{+}$, then $k$ is a zero of the functions $D(i k)$ and $D(k)$ of the same multiplicity. Due to the identity (4.9), det $S(k)$ is continuous at the point $k \in \sigma_{d}$. Therefore, $\operatorname{det} S(k)$ is continuous in $\mathbb{R}_{+}$. 
4.3. Asymptotics of $\Omega$. We consider the matrix-valued function $\Omega=\mathbb{1}_{4}+c_{k}\left(\Omega_{0}-\Omega_{1}\right)$. Substituting the definitions (3.4) into (4.7), (4.8) we obtain

$$
\begin{array}{r}
\Omega_{0}(k)=\Psi_{1}(k) \Psi_{2}(k)=\left(\begin{array}{c}
\psi_{1}(i k) \\
\psi_{1}(k)
\end{array}\right)\left(i \psi_{2}(i k), \psi_{2}(k)\right), \\
\Omega_{1}(k)=\Psi_{1}(k) Y(k) \Psi_{2}(k)=\left(\begin{array}{c}
\psi_{1}(i k) \\
\psi_{1}(k)
\end{array}\right) Y(k)\left(i \psi_{2}(i k), \psi_{2}(k)\right) .
\end{array}
$$

Introduce entire $2 \times 2$ matrix-valued functions

$$
\mathscr{B}_{1}(k)=\psi_{1}(i k) \psi_{2}(k), \quad \mathscr{B}_{2}(k)=i \psi_{1}(k) \psi_{2}(i k),
$$

The identities (4.11) and the definitions (3.13) (4.12) give

$$
\begin{gathered}
\Omega_{0}(k)=\left(\begin{array}{cc}
i \mathcal{A}_{0}(i k) & \mathscr{B}_{1}(k) \\
\mathscr{B}_{2}(k) & \mathcal{A}_{0}(k)
\end{array}\right), \\
\Omega_{1}(k)=\left(\begin{array}{cc}
i \psi_{1}(i k) Y(k) \psi_{2}(i k) & \psi_{1}(i k) Y(k) \psi_{2}(k) \\
i \psi_{1}(k) Y(k) \psi_{2}(i k) & \mathcal{A}_{1}(k)
\end{array}\right) .
\end{gathered}
$$

Introduce the domain

$$
\mathbb{K}_{1}^{+}=\left\{k \in \mathbb{K}_{1}: \arg k \in\left(0, \frac{\pi}{4}\right)\right\}
$$

Lemma 4.4. Let $p, q \in \mathcal{H}_{0}$. Then the functions $\mathscr{B}_{1}(k), \mathscr{B}_{2}(k)$, given by (4.12), satisfy

$$
\begin{gathered}
\mathscr{B}_{1}(k)=\frac{1}{2 \pi}\left(\begin{array}{cc}
\beta_{1}(k) & \beta_{2}(k) \\
\beta_{2}(-k) & \beta_{1}(-k)
\end{array}\right), \\
\mathscr{B}_{2}(k)=\frac{1}{2 \pi}\left(\begin{array}{ll}
i \beta_{1}(-k) & i \beta_{2}(k) \\
i \beta_{2}(-k) & i \beta_{1}(k)
\end{array}\right),
\end{gathered}
$$

where

$$
\beta_{1}(k)=\int_{\mathbb{R}}\left(q(x)-i 2 k^{2} p(x)\right) e^{(1+i) k x} d x, \quad \beta_{2}(k)=\int_{\mathbb{R}}\left(q(x)+i 2 k^{2} p(x)\right) e^{(1-i) k x} d x .
$$

Moreover, the function $\operatorname{det} \Omega(k)$ satisfies

$$
\operatorname{det} \Omega(k)=e^{2 \gamma(\operatorname{Re} k+\operatorname{Im} k)} O\left(k^{-1}\right),
$$

as $|k| \rightarrow \infty, k \in \overline{\mathbb{K}}_{1}^{+}$, uniformly in $\arg k \in\left[0, \frac{\pi}{4}\right]$.

Proof. Substituting the definitions (3.2), (3.3) into (4.12) we obtain the identities (4.15).

Let $k \in \overline{\mathbb{K}}_{1}^{+},|k| \rightarrow \infty$. The definitions (4.16) give

$$
\begin{array}{ll}
\beta_{1}(k)=e^{(\operatorname{Re} k-\operatorname{Im} k) \gamma} O\left(k^{2}\right), & \beta_{1}(-k)=O\left(k^{2}\right), \\
\beta_{2}(k)=e^{(\operatorname{Re} k+\operatorname{Im} k) \gamma} O\left(k^{2}\right), & \beta_{2}(-k)=O\left(k^{2}\right) .
\end{array}
$$

Substituting these asymptotics into the identities (4.15) we obtain

$$
\begin{array}{r}
\mathscr{B}_{1}(k)=\left(\begin{array}{cc}
e^{(\operatorname{Re} k-\operatorname{Im} k) \gamma} O\left(k^{2}\right) & e^{(\operatorname{Re} k+\operatorname{Im} k) \gamma} O\left(k^{2}\right) \\
O\left(k^{2}\right) & O\left(k^{2}\right)
\end{array}\right), \\
\mathscr{B}_{2}(k)=\left(\begin{array}{ll}
O\left(k^{2}\right) & e^{(\operatorname{Re} k+\operatorname{Im} k) \gamma} O\left(k^{2}\right) \\
O\left(k^{2}\right) & e^{(\operatorname{Re} k-\operatorname{Im} k) \gamma} O\left(k^{2}\right)
\end{array}\right) .
\end{array}
$$


Substituting these asymptotics and (3.16) into the identity (4.7) we obtain

$$
\Omega_{0}(k)=\left(\begin{array}{cccc}
O\left(k^{2}\right) & e^{2 \gamma \operatorname{Re} k} O\left(k^{2}\right) & e^{(\operatorname{Re} k-\operatorname{Im} k) \gamma} O\left(k^{2}\right) & e^{(\operatorname{Re} k+\operatorname{Im} k) \gamma} O\left(k^{2}\right) \\
O\left(k^{2}\right) & O\left(k^{2}\right) & O\left(k^{2}\right) & O\left(k^{2}\right) \\
O\left(k^{2}\right) & e^{(\operatorname{Re} k+\operatorname{Im} k) \gamma} O\left(k^{2}\right) & O\left(k^{2}\right) & e^{2 \gamma \operatorname{Im} k} O\left(k^{2}\right) \\
O\left(k^{2}\right) & e^{(\operatorname{Re} k-\operatorname{Im} k) \gamma} O\left(k^{2}\right) & o\left(k^{2}\right) & O\left(k^{2}\right)
\end{array}\right) .
$$

Let $|k| \rightarrow \infty, k \in \overline{\mathbb{K}}_{1}$. The estimates (3.5) and (2.23) give

$$
\begin{array}{r}
\psi_{1}(i k) Y(k) \psi_{2}(i k)=\left(\begin{array}{cc}
e^{\gamma \operatorname{Re} k} O(k) & e^{2 \gamma \operatorname{Re} k} O(k) \\
O(k) & e^{\gamma \operatorname{Re} k} O(k)
\end{array}\right), \\
\psi_{1}(i k) Y(k) \psi_{2}(k)=\left(\begin{array}{cc}
e^{\gamma \operatorname{Re} k} O(k) & e^{\gamma(\operatorname{Re} k+\operatorname{Im} k)} O(k) \\
O(k) & e^{\gamma \operatorname{Im} k} O(k)
\end{array}\right), \\
\psi_{1}(k) Y(k) \psi_{2}(i k)=\left(\begin{array}{cc}
e^{\gamma \operatorname{Im} k} O(k) & e^{\gamma(\operatorname{Re} k+\operatorname{Im} k)} O(k) \\
O(k) & e^{\gamma \operatorname{Re} k} O(k)
\end{array}\right) .
\end{array}
$$

Substituting these asymptotics and (3.17) into the identity (4.14) we obtain

$$
\Omega_{1}(k)=\left(\begin{array}{cccc}
e^{\gamma \operatorname{Re} k} O(k) & e^{2 \gamma \operatorname{Re} k} O(k) & e^{\gamma \operatorname{Re} k} O(k) & e^{\gamma(\operatorname{Re} k+\operatorname{Im} k)} O(k) \\
O(k) & e^{\gamma \operatorname{Re} k} O(k) & O(k) & e^{\gamma \operatorname{Im} k} O(k) \\
e^{\gamma \operatorname{Im} k} O(k) & e^{\gamma(\operatorname{Re} k+\operatorname{Im} k)} O(k) & e^{\gamma \operatorname{Im} k} O(k) & e^{2 \gamma \operatorname{Im} k} O(k) \\
O(k) & e^{\gamma \operatorname{Re} k} O(k) & O(k) & e^{\gamma \operatorname{Im} k} O(k)
\end{array}\right) .
$$

Substituting the asymptotics (4.18), (4.19) into the definition (3.2) we obtain the asymptotics

$$
\Omega(k)=\left(\begin{array}{cccc}
e^{\gamma \operatorname{Re} k} O\left(k^{-1}\right) & e^{2 \gamma \operatorname{Re} k} O\left(k^{-1}\right) & e^{\gamma \operatorname{Re} k} O\left(k^{-1}\right) & e^{\gamma(\operatorname{Re} k+\operatorname{Im} k)} O\left(k^{-1}\right) \\
O\left(k^{-1}\right) & e^{\gamma \operatorname{Re} k} O\left(k^{-1}\right) & O\left(k^{-1}\right) & e^{\gamma \operatorname{Im} k} O\left(k^{-1}\right) \\
e^{\gamma \operatorname{Im} k} O\left(k^{-1}\right) & e^{\gamma(\operatorname{Re} k+\operatorname{Im} k)} O\left(k^{-1}\right) & 1+e^{\gamma \operatorname{Im} k} O\left(k^{-1}\right) & e^{2 \gamma \operatorname{Im} k} O\left(k^{-1}\right) \\
O\left(k^{-1}\right) & e^{\gamma \operatorname{Re} k} O\left(k^{-1}\right) & O\left(k^{-1}\right) & 1+e^{\gamma \operatorname{Im} k} O\left(k^{-1}\right)
\end{array}\right)
$$

as $|k| \rightarrow \infty, k \in \overline{\mathbb{K}}_{1}^{+}$, which yields (4.17).

\section{Proof of the main Theorems}

5.1. Asymptotics of the determinant. We prove our preliminary Proposition 1.1.

Proof of Proposition 1.1. i) The statement is proved in Lemma 2.1 i).

ii) Due to Lemma 4.1, the function $D(k)$ has an analytic extension from $\mathbb{K}_{1}$ onto $\mathbb{C} \backslash\{0\}$, it is real on the line $e^{i \frac{\pi}{4}} \mathbb{R}$ and the function $k^{4} D(k)$ is entire. The asymptotics (4.4) yields the asymptotics (1.7). This asymptotics shows that the function $D(k)$ has a finite number of zeros in $\overline{\mathbb{K}}_{1}$. Then the operator $H$ has a finite number of eigenvalues.

We determine asymptotics of the determinant in the complex plane. Due to the symmetry of $D(k)$ we need to get this asymptotics in the domains $\mathbb{K}_{1}, \mathbb{K}_{2}, \mathbb{K}_{3}$. The asymptotics in the domain $\mathbb{K}_{1}$ is known due to (4.4). We analyze the function $D(k)$ in the domains $\mathbb{K}_{2}, \mathbb{K}_{3}$ by the following way. We obtain the asymptotics of $S(k)$ and $\Omega(k)$ in $\mathbb{K}_{1}$. Then we use the identities (4.9), (4.10) in order to determine the asymptotics of $D(i k), D(-k)$ in $\mathbb{K}_{1}$, which gives the asymptotics of $D(k)$ in $\mathbb{K}_{2}, \mathbb{K}_{3}$. 
Lemma 5.1. Let $p, q \in \mathcal{H}_{0}$. Then

$$
\begin{gathered}
\operatorname{det} S(k)=1+O\left(k^{-1}\right)+e^{2 \gamma \operatorname{Im} k} O\left(k^{-2}\right), \\
D(i k)=1+O\left(k^{-1}\right)+e^{2 \gamma \operatorname{Im} k} O\left(k^{-2}\right),
\end{gathered}
$$

as $k \in \overline{\mathbb{K}}_{1},|k| \rightarrow \infty$, uniformly in $\arg k \in\left[0, \frac{\pi}{2}\right]$,

$$
D(-k)=1+e^{2 \gamma(\operatorname{Re} k+\operatorname{Im} k)} O\left(k^{-1}\right)
$$

as $k \in \overline{\mathbb{K}}_{1}^{+},|k| \rightarrow \infty$, uniformly in $\arg k \in\left[0, \frac{\pi}{4}\right]$.

Proof. Let $|k| \rightarrow \infty, k \in \overline{\mathbb{K}}_{1}$. Substituting the asymptotics (3.16) and (3.17) into the identity (3.11) we obtain the asymptotics

$$
S(k)=\left(\begin{array}{cc}
1+O\left(k^{-1}\right)+e^{\gamma \operatorname{Im} k} O\left(k^{-2}\right) & e^{2 \gamma \operatorname{Im} k} O\left(k^{-1}\right) \\
o\left(k^{-1}\right) & 1+O\left(k^{-1}\right)+e^{\gamma \operatorname{Im} k} O\left(k^{-2}\right)
\end{array}\right),
$$

which yields the asymptotics (5.1). Substituting the asymptotics (1.7) and (5.1) into (4.9) we obtain the asymptotics (5.2).

Substituting the asymptotics (1.7), (4.17) into the identity (4.10) we obtain (5.3).

We prove Theorem 1.2

Proof of Theorem 1.2. The asymptotics (1.7) gives the estimate (1.8) in $\mathbb{K}_{1}$, the asymptotics (5.2) gives (1.8) in $\mathbb{K}_{2}$, the asymptotics (5.3) gives (1.8) in $\mathbb{K}_{3}$. The estimate (1.8) in $\mathbb{K}_{2}$ and the symmetry $D(k)=\overline{D(i \bar{k})}$ imply the estimate $(1.8)$ in $\mathbb{K}_{4}$.

The asymptotics (5.2) yields $|k(D(k)-1)| \leqslant C e^{-2 \gamma \operatorname{Re} k}$ for all $k \in \mathbb{K}_{2}$ for some $C>0$. Let $k_{*} \in \mathbb{K}_{2}$ be a resonance. Then the identity $D\left(k_{*}\right)=0$ gives the estimate (1.10).

We prove the estimate (1.9). Recall that the function $D(k)$ is analytic in $\mathbb{C} \backslash\{0\}$ and may have a pole of order $\leqslant 4$ at the point $k=0$. Let the function $F(k)=k^{m} D(k), m \leqslant 4$, be entire and satisfy $F(0) \neq 0$. Let $\mathcal{N}_{F}(r)$ be the number of zeros of the function $F$ in the disc $|k|<r$ counted with multiplicity. If $D(0) \neq 0$, then $\mathcal{N}=\mathcal{N}_{F}$, if $k=0$ is a zero of $D$ of multiplicity $\ell$, then $\mathcal{N}=\mathcal{N}_{F}+\ell$. We have to prove that $\mathcal{N}_{F}$ satisfies the estimate (1.9). The estimate (1.8) gives

$$
\log |F(k)| \leqslant 2 \gamma\left((\operatorname{Re} k)_{-}+(\operatorname{Im} k)_{-}\right)+C \log |k|
$$

for all $k \in \mathbb{C},|k|$ large enough and for some $C>0$. Substituting the estimate (5.4) into Jensen's formula

$$
\int_{0}^{r} \frac{\mathcal{N}_{F}(t)}{t} d t=\frac{1}{2 \pi} \int_{0}^{2 \pi} \log \left|F\left(r e^{i \theta}\right)\right| d \theta-\log |F(0)|,
$$

we obtain

$$
\int_{0}^{r} \frac{\mathcal{N}_{F}(t)}{t} d t \leqslant-\frac{\gamma r}{\pi}\left(\int_{\frac{\pi}{2}}^{\pi} \cos \theta d \theta+\int_{\pi}^{\frac{3 \pi}{2}}(\cos \theta+\sin \theta) d \theta+\int_{\frac{3 \pi}{2}}^{2 \pi} \sin \theta d \theta\right)+C \log r=\frac{4 \gamma r}{\pi}+C \log r
$$

for all $r>0$ large enough. Then there exists

$$
\lim _{r \rightarrow+\infty} \frac{1}{r} \int_{0}^{r} \frac{\mathcal{N}_{F}(t)}{t} d t \leqslant \frac{4 \gamma}{\pi}
$$

The estimate (1.9) follows from the following well known result, see, e.g., [Le96, Lm II.4.3]: 
Let $\mathcal{N}_{F}(t)$ be non-decreasing function on $\mathbb{R}_{+}, \mathcal{N}_{F}(t)=0$ as $0 \leqslant t<\varepsilon$ for some $\varepsilon>0$, and let the function

$$
I(r)=\frac{1}{r} \int_{0}^{r} \frac{\mathcal{N}_{F}(t)}{t} d t, \quad r \in \mathbb{R}_{+},
$$

has the limit as $r \rightarrow \infty$. Then $\mathcal{N}_{F}(r)=r(I(r)+o(1))$ as $r \rightarrow \infty$.

5.2. Trace formulas. Let $\zeta_{n}, n \in \mathbb{N}$, be the zeros of the function $D$ in $\mathbb{C} \backslash\{0\}$ labeled by $0<\left|\zeta_{1}\right| \leqslant\left|\zeta_{2}\right| \leqslant \ldots$ counting with multiplicities. The estimate (1.8) provides the standard Hadamard factorization

$$
D(k)=\frac{\alpha}{k^{m}} e^{\beta k} \lim _{r \rightarrow \infty} \prod_{\left|\zeta_{n}\right|<r}\left(1-\frac{k}{\zeta_{n}}\right) e^{\frac{k}{\zeta_{n}}}, \quad m \leqslant 4,
$$

absolutely and uniformly on any compact subset in $\mathbb{C} \backslash\{0\}$, where $\alpha, \beta$ are defined in (1.15). The identity (5.6) gives

$$
\frac{D^{\prime}(k)}{D(k)}=\beta-\frac{m}{k}+k \lim _{r \rightarrow \infty} \sum_{\left|\zeta_{n}\right|<r} \frac{1}{\zeta_{n}\left(k-\zeta_{n}\right)} .
$$

The following proofs use the approach from [K04.

Proof of Theorem 1.4. Let $k \in \mathbb{K}_{1} \backslash \sigma_{d}$. The definitions (1.3), (2.12) show that the operators $V R_{0}(k), V_{2} R_{0}(k)$ and $R_{0}(k) V_{1}$ are Hilbert-Schmidt. Then the operator

$$
R_{0}(k)-R(k)=R_{0}(k) V R(k)=R_{0}(k) V R_{0}(k)-R_{0}(k) V R_{0}(k) V R(k)
$$

is trace class. Due to the identities (2.2), (2.4), (2.20) and $Y_{0}^{\prime}(k)=4 k^{3} V_{2} R_{0}^{2}(k) V_{1}$, the derivative of $D$ satisfies

$$
\frac{1}{4 k^{3}} \frac{D^{\prime}(k)}{D(k)}=\operatorname{Tr}\left(\left(I+Y_{0}(k)\right)^{-1} V_{2} R_{0}^{2}(k) V_{1}\right)=\operatorname{Tr} R_{0}(k) V R(k)=\operatorname{Tr}\left(R_{0}(k)-R(k)\right) .
$$

The identity (5.7) together with (15.8) yields the trace formula (1.16).

The S-matrix $S(k), k \in \mathbb{R}_{+}$, is a complex $2 \times 2$ matrix and $|\operatorname{det} S(k)|=1$. Thus we have

$$
\operatorname{det} S(k)=e^{-2 \pi i \phi_{s c}(k)}, \quad k \in \mathbb{R}_{+} .
$$

Since $\operatorname{det} S(k)$ is continuous in $\mathbb{R}_{+}$and $\operatorname{det} S(k)=1+O\left(k^{-1}\right)$ as $k \rightarrow+\infty$ (see (5.1)), formula (5.9) determines $\phi_{s c}(k)$ by the identity $\phi_{s c}(k)=\frac{i}{2 \pi} \log \operatorname{det} S(k)$, the continuity, and the asymptotics $\phi_{s c}(k)=O\left(k^{-1}\right)$ as $k \rightarrow+\infty$.

Theorem 5.2. Let $(p, q) \in \mathcal{H}_{0}$. Then

$$
\phi_{s c}^{\prime}(k)=\frac{1}{2 \pi i}\left((1-i) \beta+\sum_{n=1}^{\infty} \frac{k}{\zeta_{n}}\left(\frac{1}{i k-\zeta_{n}}+\frac{1}{k-\zeta_{n}}\right)\right), \quad k \in \mathbb{R}_{+} \backslash \sigma_{d},
$$

the series converges absolutely and uniformly on any compact subset in $\mathbb{R}_{+} \backslash \sigma_{d}$.

Proof. The function $\operatorname{det} S(k)$ is continuous in $\mathbb{R}_{+}$, has a meromorphic extension onto the whole complex plane and, due to equations (5.9) and (4.9), it satisfies the identities

$$
e^{-2 \pi i \phi_{s c}(k)}=\operatorname{det} S(k)=\frac{D(i k)}{D(k)}, \quad \forall \quad k>0 .
$$


Differentiating this identity we obtain

Then the identity (5.7) implies (5.10).

$$
-2 \pi i \phi_{s c}^{\prime}(k)=i \frac{D^{\prime}(i k)}{D(i k)}-\frac{D^{\prime}(k)}{D(k)} .
$$

5.3. The Euler-Bernoulli operator. We consider the Euler-Bernoulli operator

$$
\mathcal{E} u=\frac{1}{b}\left(a u^{\prime \prime}\right)^{\prime \prime}
$$

acting on $L^{2}(\mathbb{R}, b(x) d x)$, where the coefficients $a, b$ satisfy

$$
a>0, \quad b>0, \quad a-1, b-1 \in \mathcal{H}_{4}(1) .
$$

Now we consider the Liouville type transformation of the operator $\mathcal{E}$ into the operator $H$, defined by (1.1) with specific $p, q$ depending on $a, b$. In order to define this transformation we introduce the new variable $t \in \mathbb{R}$ by

$$
t=t(x)=\int_{0}^{x}\left(\frac{b(s)}{a(s)}\right)^{\frac{1}{4}} d s, \quad \forall \quad x \in \mathbb{R} .
$$

Let $x=x(t)$ be the inverse function for $t(x), x \in \mathbb{R}$. Introduce the unitary transformation $U: L^{2}(\mathbb{R}, b(x) d x) \rightarrow L^{2}(\mathbb{R}, d t)$ by

$$
u(x) \mapsto y(t)=(U u)(t)=a^{\frac{1}{8}}(x(t)) b^{\frac{3}{8}}(x(t)) u(x(t)) \quad \forall \quad t \in \mathbb{R} .
$$

Introduce the functions $\alpha(t), \beta(t), t \in \mathbb{R}$, by

$$
\alpha(t)=\frac{1}{a(x(t))} \frac{d a(x(t))}{d t}, \quad \beta(t)=\frac{1}{b(x(t))} \frac{d b(x(t))}{d t} .
$$

Then the functions $\alpha, \beta \in L^{1}(\mathbb{R})$ are real, compactly supported and satisfy

$$
\dddot{a}, \dddot{\beta} \in L^{1}(\mathbb{R}), \quad \text { where } \quad \dot{f}=\frac{d f}{d t} .
$$

Let the operator $H$ be defined by (1.1), where the coefficients $p(t), q(t), t \in \mathbb{R}$, have the forms

$$
\begin{gathered}
p=-\frac{\dot{\eta}_{0}+\varkappa}{2}, \\
q=\frac{d}{d t}\left(\left(\dot{\eta}_{2}+\eta_{2}^{2}\right) \eta_{1}-\ddot{\eta}_{1}\right)+\left(\left(\dot{\eta}_{2}+\eta_{2}^{2}\right) \eta_{1}-\ddot{\eta}_{1}\right) \eta_{1},
\end{gathered}
$$

and the functions $\varkappa(t), \eta_{0}(t), \eta_{1}(t), \eta_{2}(t)$ are given by

$$
\begin{gathered}
\varkappa=\frac{5 \alpha^{2}+5 \beta^{2}+6 \alpha \beta}{32} \geqslant \frac{\alpha^{2}+\beta^{2}}{16} \geqslant 0, \\
\eta_{0}=\frac{3 \alpha+5 \beta}{4}, \quad \eta_{1}=\frac{\alpha+3 \beta}{8}, \quad \eta_{2}=\frac{3 \alpha+\beta}{8} .
\end{gathered}
$$

The coefficients satisfy: $p, p^{\prime \prime}, q \in L^{1}\left(\mathbb{R}_{+}\right),(p, q) \in \mathcal{H}_{2} \times \mathcal{H}_{0}$ with $\gamma$ given by (1.17).

Let the coefficients $a, b$ satisfy the conditions (5.12). Let the operator $\mathcal{E}$ be defined by (5.11) and let the operator $H$ be defined by (1.1), where the coefficients $p, q$ have the forms (5.16), (5.17). Repeating the arguments from [BK15] we obtain that the operators $\mathcal{E}$ and $H$ are unitarily equivalent and satisfy:

$$
\mathcal{E}=U^{-1} H U
$$


where the operator $U$ is defined by (15.14).

Corollary 5.3. Let $a-1, b-1 \in \mathcal{H}_{4}(1)$ and let $a, b$ be positive. Then the determinant $D(k)$ satisfies

$$
D(k)=1+\frac{1+i}{4 k} \int_{\mathbb{R}} \varkappa(t) d t+\frac{O(1)}{k^{2}} \quad \text { as } \quad|k| \rightarrow \infty, \quad k \in \overline{\mathbb{K}}_{1}
$$

uniformly in $\arg k \in\left[0, \frac{\pi}{2}\right]$, where $\varkappa(t)$ is given by the definition (5.18).

Proof. Identity (5.16) gives

$$
p_{0}=\int_{\mathbb{R}} p(t) d t=-\frac{1}{2} \int_{\mathbb{R}} \varkappa(t) d t
$$

Substituting this identities into the asymptotics (1.7) we obtain the asymptotics (5.20).

The definition (5.18) shows that $\varkappa \geqslant 0$, moreover, $\varkappa=0$ iff $\alpha=\beta=0$. Then the second term in the asymptotics (5.20) vanishes iff $\alpha=\beta=0$. The proof of Theorem 1.6 is based on this observation.

Proof of Theorem 1.6. Assume that the operator $\mathcal{E}$ does not have any eigenvalues and resonances. Then $D=1$ and the second term in the asymptotics (5.20) vanishes. The estimates (5.18) show that $\alpha=\beta=0$ in this case, then $a=b=1$ on $\mathbb{R}$.

Conversely, assume that $a=b=1$ on $\mathbb{R}$. Then $\alpha=\beta=0$ and the definitions (5.16), (5.17) imply $p=q=0$. The identities (1.3) yield $V_{1}=0, V_{2}=0$. Then the definition (1.4) gives $Y_{0}=0$, and the identity (1.5) implies $D=1$. Therefore, there are not any eigenvalues and resonances.

\section{Asymptotics of the Resonances}

6.1. Asymptotics of the determinant. The function $D(k)$ has a finite number of zeros in the domain $\mathbb{K}_{1}$. The identity (4.9) shows that $i k$ with large $|k|$ is a resonance in $\mathbb{K}_{2}$ iff $k$ is a zero of the function $\operatorname{det} S(k)$ in $\mathbb{K}_{1}$. Thus in order to determine asymptotics of resonances in $\mathbb{K}_{2}$ we need to improve asymptotics of $\operatorname{det} S(k)$ in $\mathbb{K}_{1}$. Similarly, the identity (4.10) shows that $-k$ with large $|k|$ is a resonance in $\mathbb{K}_{3}$ iff $k$ is a zero of the function $\operatorname{det} \Omega(k)$ in $\mathbb{K}_{1}$. Then in order to determine asymptotics of resonances in $\mathbb{K}_{3}$ we have to improve asymptotics of the function det $\Omega(k)$ in $\mathbb{K}_{1}$. Moreover, due to the symmetry of the determinant it is sufficiently to consider in this case the domain

$$
\mathbb{K}_{1}^{+}=\left\{k \in \mathbb{C}: \arg k \in\left(0, \frac{\pi}{4}\right)\right\} .
$$

Lemma 6.1. Let $p, q$ satisfy the conditions (1.12). Then the S-matrix $S(k)$ and the matrixvalued function $\Omega(k)$, defined by (4.6), satisfy

$$
\operatorname{det} S(k)=1-\frac{e^{-i 2 k \gamma} p_{+} p_{-}}{(2 k)^{4}}\left(1+o(1)+e^{-\gamma \operatorname{Im} k} O(k)\right)+O\left(k^{-1}\right),
$$

as $|k| \rightarrow \infty, k \in \overline{\mathbb{K}}_{1}$, uniformly in $\arg k \in\left[0, \frac{\pi}{2}\right]$,

$$
\operatorname{det} \Omega(k)=\frac{e^{2(1-i) k \gamma}\left(p_{+} p_{-}\right)^{2}}{(2 k)^{8}}\left(1+o(1)+e^{-\gamma \operatorname{Im} k} O(k)\right)-\frac{e^{2 k \gamma} p_{+} p_{-}}{(2 k)^{4}}(1+o(1)),
$$

as $|k| \rightarrow \infty, k \in \overline{\mathbb{K}}_{1}^{+}$, uniformly in $\arg k \in\left[0, \frac{\pi}{4}\right]$. 
Proof. Let $k \in \overline{\mathbb{K}}_{1},|k| \rightarrow \infty$. The definition (3.11) and the asymptotics (3.18) and (3.20) give

$$
S(k)=\mathbb{1}_{2}+c_{k}\left(\mathcal{A}_{0}(k)-\mathcal{A}_{1}(k)\right)=\frac{1}{4 k^{2}} E_{1}^{+}(k) G_{1}(k) E_{1}^{-}(k),
$$

where

$$
\begin{gathered}
c_{k}=\frac{\pi}{i 2 k^{3}}, \quad E_{1}^{+}(k)=\left(\begin{array}{cc}
e^{-i \gamma k} & 0 \\
0 & 1
\end{array}\right), \quad E_{1}^{-}(k)=\left(\begin{array}{cc}
1 & 0 \\
0 & e^{-i \gamma k}
\end{array}\right), \\
G_{1}(k)=\left(\begin{array}{cc}
4 k^{2} e^{i k \gamma}\left(1+O\left(k^{-1}\right)\right)+O\left(k^{-1}\right) & p_{+}+o(1)+e^{-2 \gamma \operatorname{Im} k} O(1) \\
p_{-}+o(1)+e^{-2 \gamma \operatorname{Im} k} O(1) & 4 k^{2} e^{i k \gamma}\left(1+O\left(k^{-1}\right)\right)+O\left(k^{-1}\right)
\end{array}\right) .
\end{gathered}
$$

The asymptotics (6.5) implies

$$
\operatorname{det} G_{1}(k)=16 k^{4} e^{2 i k \gamma}\left(1+O\left(k^{-1}\right)\right)-p_{+} p_{-}+o(1)+e^{-\gamma \operatorname{Im} k} O(k) .
$$

The identity (6.3) and the definitions (6.4) yield the asymptotics (6.1).

We prove the asymptotics (6.2). Substituting the identities (4.13), (4.14) into the definition (4.6) and using the definition (3.11) we obtain

$$
\Omega(k)=\left(\begin{array}{cc}
\mathbb{1}_{2}+c_{k}\left(i \mathcal{A}_{0}(i k)-i \psi_{1}(i k) Y(k) \psi_{2}(i k)\right) & c_{k}\left(\mathscr{B}_{1}(k)-\psi_{1}(i k) Y(k) \psi_{2}(k)\right) \\
c_{k}\left(\mathscr{B}_{2}(k)-i \psi_{1}(k) Y(k) \psi_{2}(i k)\right) & S(k)
\end{array}\right) .
$$

Let $k \in \overline{\mathbb{K}}_{1}^{+},|k| \rightarrow \infty$ and let $p_{+} p_{-} \neq 0$. Integrations by parts in the definitions (4.16) give

$$
\begin{array}{r}
\beta_{1}(k)=e^{(\operatorname{Re} k-\operatorname{Im} k) \gamma} O(k), \quad \beta_{1}(-k)=O(k), \\
\beta_{2}(k)=-(1-i) k e^{(1-i) k \gamma}\left(p_{+}+o(1)\right), \quad \beta_{2}(-k)=-(1-i) k\left(p_{-}+o(1)\right) .
\end{array}
$$

Substituting these asymptotics into the identities (4.15) we obtain

$$
\begin{gathered}
\mathscr{B}_{1}(k)=-\frac{k}{2 \pi}\left(\begin{array}{cc}
e^{(\operatorname{Re} k-\operatorname{Im} k) \gamma} O(1) & \left.(1-i) e^{(1-i) k \gamma}\left(p_{+}+o(1)\right)\right) \\
(1-i)\left(p_{-}+o(1)\right) & O(1)
\end{array}\right), \\
\mathscr{B}_{2}(k)=-\frac{k}{2 \pi}\left(\begin{array}{cc}
O(1) & (1+i) e^{(1-i) k \gamma}\left(p_{+}+o(1)\right) \\
(1+i) p_{-}+o(1) & e^{(\operatorname{Re} k-\operatorname{Im} k) \gamma} O(1)
\end{array}\right) .
\end{gathered}
$$

Repeating the arguments from the proof of the asymptotics (3.20) we obtain

$$
\begin{gathered}
\psi_{1}(i k) Y(k) \psi_{2}(i k)=\left(\begin{array}{cc}
e^{\gamma \operatorname{Re} k} O(1) & e^{2 \gamma \operatorname{Re} k} O(1) \\
O(1) & e^{\gamma \operatorname{Re} k} O(1)
\end{array}\right), \\
\psi_{1}(i k) Y(k) \psi_{2}(k)=\left(\begin{array}{cc}
e^{\gamma \operatorname{Re} k} O(1) & e^{\gamma(\operatorname{Re} k+\operatorname{Im} k)} O(1) \\
O(1) & e^{\gamma \operatorname{Im} k} O(1)
\end{array}\right), \\
\psi_{1}(k) Y(k) \psi_{2}(i k)=\left(\begin{array}{cc}
e^{\gamma \operatorname{Im} k} O(1) & e^{\gamma(\operatorname{Re} k+\operatorname{Im} k)} O(1) \\
O(1) & e^{\gamma \operatorname{Re} k} O(1)
\end{array}\right) .
\end{gathered}
$$

The asymptotics (3.19) and (6.9) give

$$
\mathbb{1}_{2}+c_{k}\left(i \mathcal{A}_{0}(i k)-i \psi_{1}(i k) Y(k) \psi_{2}(i k)=\frac{1}{4 k^{2}} E_{2}^{+}(k) G_{2}(k) E_{2}^{-}(k),\right.
$$

where

$$
\begin{gathered}
E_{2}^{+}(k)=\left(\begin{array}{cc}
e^{\gamma k} & 0 \\
0 & 1
\end{array}\right), \quad E_{2}^{-}(k)=\left(\begin{array}{cc}
1 & 0 \\
0 & e^{\gamma k}
\end{array}\right), \\
G_{2}(k)=\left(\begin{array}{cc}
O\left(k^{-1}\right) & -p_{+}+o(1) \\
-p_{-}+o(1) & O\left(k^{-1}\right)
\end{array}\right) .
\end{gathered}
$$


The asymptotics (6.7) and (6.10) imply

where

$$
c_{k}\left(\mathscr{B}_{1}(k)-\psi_{1}(i k) Y(k) \psi_{2}(k)\right)=\frac{1}{4 k^{2}} E_{2}^{+}(k) G_{3}(k) E_{1}^{-}(k),
$$

$$
G_{3}(k)=\left(\begin{array}{cc}
O\left(k^{-1}\right)+e^{-\gamma \operatorname{Im} k} O(1) & (1+i) p_{+}+o(1) \\
(1+i) p_{-}+o(1) & O\left(k^{-1}\right)+e^{-\gamma \operatorname{Im} k} O(1)
\end{array}\right) .
$$

The asymptotics (6.8) and (6.11) yield

$$
c_{k}\left(\mathscr{B}_{2}(k)-i \psi_{1}(k) Y(k) \psi_{2}(i k)\right)=\frac{1}{4 k^{2}} E_{1}^{+}(k) G_{4}(k) E_{2}^{-}(k),
$$

where

$$
G_{4}(k)=\left(\begin{array}{cc}
O\left(k^{-1}\right)+e^{-\gamma \operatorname{Im} k} O(1) & i(1+i) p_{+}(1+o(1)) \\
i(1+i) p_{-}(1+o(1)) & O\left(k^{-1}\right)+e^{-\gamma \operatorname{Im} k} O(1)
\end{array}\right) .
$$

Substituting the identities (6.3), (6.12), (6.15) and (6.17) into the relation (6.6) we obtain the identity

$$
\Omega(k)=\frac{1}{4 k^{2}}\left(\begin{array}{cc}
E_{2}^{+}(k) & 0 \\
0 & E_{1}^{+}(k)
\end{array}\right)\left(\begin{array}{cc}
G_{2}(k) & G_{3}(k) \\
G_{4}(k) & G_{1}(k)
\end{array}\right)\left(\begin{array}{cc}
E_{2}^{-}(k) & 0 \\
0 & E_{1}^{-}(k)
\end{array}\right) .
$$

The definitions (6.4), (6.13) and the identity (6.19) yield

$$
\operatorname{det} \Omega(k)=\frac{e^{2(1-i) k \gamma}}{(2 k)^{8}} \operatorname{det}\left(\begin{array}{ll}
G_{2}(k) & G_{3}(k) \\
G_{4}(k) & G_{1}(k)
\end{array}\right) .
$$

The standard matrix formula gives

$$
\operatorname{det}\left(\begin{array}{ll}
G_{2}(k) & G_{3}(k) \\
G_{4}(k) & G_{1}(k)
\end{array}\right)=\operatorname{det} G_{2}(k) \operatorname{det} G(k),
$$

where

$$
G(k)=G_{1}(k)-G_{4}(k) G_{2}^{-1}(k) G_{3}(k) .
$$

Substituting this identity into (6.20) we obtain

$$
\operatorname{det} \Omega(k)=\frac{e^{2(1-i) k \gamma}}{(2 k)^{8}} \operatorname{det} G_{2}(k) \operatorname{det} G(k) .
$$

The definition (6.14) implies

$$
\operatorname{det} G_{2}(k)=-p_{+} p_{-}(1+o(1)), \quad G_{2}^{-1}(k)=\left(\begin{array}{cc}
O\left(k^{-1}\right) & -\frac{1}{p_{-}}(1+o(1)) \\
-\frac{1}{p_{+}}(1+o(1)) & O\left(k^{-1}\right)
\end{array}\right) .
$$

The asymptotics (6.5), (6.16), (6.18) and (6.22) yield

$$
\begin{gathered}
G_{4}(k) G_{2}^{-1}(k)=\left(\begin{array}{cc}
-i(1+i)+o(1) & e^{-\gamma \operatorname{Im} k} O(1)+O\left(k^{-1}\right) \\
e^{-\gamma \operatorname{Im} k} O(1)+O\left(k^{-1}\right) & -i(1+i)+o(1)
\end{array}\right), \\
G_{4}(k) G_{2}^{-1}(k) G_{3}(k)=\left(\begin{array}{cc}
e^{-\gamma \operatorname{Im} k} O(1)+O\left(k^{-1}\right) & 2 p_{+}+o(1)+e^{-2 \gamma \operatorname{Im} k} O(1) \\
2 p_{-}+o(1)+e^{-2 \gamma \operatorname{Im} k} O(1) & e^{-\gamma \operatorname{Im} k} O(1)+O\left(k^{-1}\right)
\end{array}\right), \\
G(k)=\left(\begin{array}{cc}
4 k^{2} e^{i k \gamma}\left(1+O\left(k^{-1}\right)\right)+O\left(k^{-1}\right) & -p_{+}+o(1)+e^{-2 \gamma \operatorname{Im} k} O(1) \\
-p_{-}+o(1)+e^{-2 \gamma \operatorname{Im} k} O(1) & 4 k^{2} e^{i k \gamma}\left(1+O\left(k^{-1}\right)\right)+O\left(k^{-1}\right)
\end{array}\right),
\end{gathered}
$$

which yields

$$
\operatorname{det} G(k)=16 k^{4} e^{2 i k \gamma}\left(1+O\left(k^{-1}\right)\right)-p_{+} p_{-}+o(1)+e^{-\gamma \operatorname{Im} k} O(k) .
$$


Substituting the asymptotics (6.22) and (6.23) into the identity (6.21) we obtain the asymptotics (6.2).

6.2. Asymptotics of resonances. We are ready to determine asymptotics of resonances.

Proof of Theorem 1.3. Let $k \in \mathbb{K}_{1},|k| \rightarrow \infty$ and let $i k$ be a resonance. The identity (4.9) shows that $k$ is a zero of the function $\operatorname{det} S(k)$ in $\mathbb{K}_{1}$. The asymptotics (6.1) and the identity $\operatorname{det} S(k)=0$ imply that $k$ satisfies the equation

$$
k^{4}=\frac{p_{+} p_{-}}{16} e^{-i 2 k \gamma}\left(1+o(1)+e^{-\gamma \operatorname{Im} k} O(k)\right) .
$$

Then $k$ lies on the logarithmic curve $\Gamma$ in $\mathbb{K}_{1}$, given by

$$
|k|=\frac{\left|p_{+} p_{-}\right|^{\frac{1}{4}}}{2} e^{\frac{1}{2} \gamma \operatorname{Im} k}(1+o(1)),
$$

and satisfies

$$
i k=\frac{i j_{n} \pi}{\gamma}-\frac{2 \log k}{\gamma}+\frac{1}{2 \gamma} \log \frac{\left|p_{+} p_{-}\right|}{16}+o(1)
$$

and there are not any other large resonances in $i \mathbb{K}_{+}$.

Let $k \in \mathbb{K}_{1}^{+}$, let $-k$ be a resonance and let $|k|$ be large enough. The identity (4.10) shows that $-k$ is a zero of the function $\operatorname{det} \Omega(k)$ in $\mathbb{K}_{1}$. The identity $\operatorname{det} \Omega(k)=0$ and the asymptotics (6.2) gives

$$
k^{4}=\frac{p_{+} p_{-}}{16} e^{-2 i k \gamma}\left(1+o(1)+e^{-\gamma \operatorname{Im} k} O(k)\right) .
$$

Then $k$ lies on the curve $\Gamma$ and satisfies

$$
-k=-\frac{j_{n} \pi}{\gamma}-\frac{i 2 \log k}{\gamma}+\frac{i}{2 \gamma} \log \frac{\left|p_{+} p_{-}\right|}{16}+o(1)
$$

and there are not any other large resonances in $-\mathbb{K}_{1}^{+}$. The asymptotics (6.24), (6.25) give the asymptotics (1.13), which yields the asymptotics (1.14).

The estimates (1.8) and (1.15) yield that the determinants $k^{4} D(k)$ is of exponential type. But the asymptotics (1.13) and the asymptotics of the Levinson Theorem (1.11) show that the determinants $D(k)$ is not of the Cartwright class.

6.3. Further discussions. We will discuss what properties of resonances of the second and fourth order operators with compactly supported coefficients are common and which are specific.

- Common properties:

1) The determinants $D(k)$ and $d(k)$ are exponentially type functions of the variable $k$ and each of them has an axis of symmetry.

2) For coefficients with steps the resonances have the logarithmic type asymptotics.

- Specific properties of the determinant $d(k)$ for a Schrödinger operator:

1) In terms of the spectral parameter $\lambda$ the Riemann surface for the determinant $d\left(\lambda^{\frac{1}{2}}\right)$ is the two sheeted Riemann surface for the function $\lambda^{\frac{1}{2}}$. The function $d(k) \sim 1$ as $|\lambda| \rightarrow \infty$ on the physical sheet and $d(k) \sim e^{2 \gamma|\operatorname{Im} \sqrt{\lambda}|}$ on the non-physical sheet. It has a finite number of zeros (eigenvalues) on the physical sheet and an infinite number of zeros (resonances) on the non-physical one.

2) The determinant $d(k)$ belongs to the Cartwright class $\mathcal{C}_{\text {Cart }}$. Then the Levinson Theorem gives the distribution of resonances in the large disc. 
3) The number of resonances in the disk $|\lambda|<r$ for large $r$ has asymptotics $\frac{2 \gamma}{\pi} r^{\frac{1}{2}}(1+o(1))$.

4) Using one identity (2.10) we obtain an analytic extension of the determinant from the physical sheet onto the non-physical one.

- Specific properties of the determinant $D(k)$ for a fourth order operator:

1) The Riemann surface for the determinant $D\left(\lambda^{\frac{1}{4}}\right)$ is the four sheeted Riemann surface for the function $\lambda^{\frac{1}{4}}$. The function $D$ satisfies: $D \sim 1$ at $|\lambda| \rightarrow \infty$ on the first sheet, $D \sim e^{2 \gamma|\lambda|^{\frac{1}{4}}}$ on the second and fourth sheets and $D \sim e^{2 \sqrt{2} \gamma|\lambda|^{\frac{1}{4}}}$ on the third sheet. It has a finite number of zeros (eigenvalues) on the first (physical) sheet and an infinite number of zeros (resonances) on the other (non-physical) sheets. The number of resonances in the large disc on the third sheet is, roughly speaking, in two times more than on the second (or fourth) sheet.

2) The determinant $D$ is not in the Cartwright class.

3) The number of resonances in the disk $|\lambda|<r$ has asymptotics $\frac{4 \gamma}{\pi} r^{\frac{1}{4}}(1+o(1))$ as $r \rightarrow \infty$.

4) In order to obtain an analytic extension of the determinant from the first sheet onto the other sheets we need to use two identities (4.9), (4.10).

Acknowledgments. A. Badanin was supported by the RFBR grant No 16-01-00087. E. Korotyaev was supported by the RSF grant No. 15-11-30007.

\section{REFERENCES}

[AP08] Aktosun, T., Papanicolaou, V. G. Time evolution of the scattering data for a fourth-order linear differential operator. Inverse Problems, 24(5) (2008), 055013.

[BK15] Badanin, A., Korotyaev, E. Inverse problems and sharp eigenvalue asymptotics for Euler-Bernoulli operators. Inverse Problems, 31 (2015), 055004.

[BK16] Badanin, A., Korotyaev, E. Determinant and fundamental solutions for 4-th order operators. Preprint, 2016.

[BK17] Badanin, A., Korotyaev, E. Resonances for Euler-Bernoulli operator on the half-line. Journal of Differential Equations, 263 (2017), 534-566.

[B85] Beals, R. The inverse problem for ordinary differential operators on the line. American Journal of Mathematics, 107(2) (1985), 281-366.

[BDT88] Beals, R., Deift, P., Tomei, C. Direct and inverse scattering on the line, Nathematical survays and monograph series, No. 28, AMS, Providence, 1988.

[Bu68] Butler, J. B. On the inverse problem for differential operators of fourth order with rational reflection coefficients. Journal of Differential Equations, 4(4) (1968), 573-589.

[Ko88] Koosis, P. The logarithmic integral I, Cambridge Univ. Press, Cambridge, London, New York 1988.

[DT79] Deift, P., Trubowitz, E. Inverse scattering on the line. Communications on Pure and Applied Mathematics, 32(2) (1979), 121-251.

[Fa64] Faddeev, L. D. Properties of the S-matrix of the one-dimensional Schrödinger equation, Trudy Mat. Inst. Steklov 73(1964), 314-33 (Engl. Transl. AMST 265 13966)

The inverse problem in the quantum theory of scattering. Journal of Mathematical Physics, 4.1 (1963), 72-104, translated from Usp. Mat. Nauk (1959).

[F97] Froese, R. Asymptotic distribution of resonances in one dimension. Journal of differential equations, 137 (1997), 251-272.

[Ga28] Gamow, G. Zur Quantentheorie des Atomkernes, Zeitschrift fur Physik, 51(1928), no 3-4, 204-212.

[Gr75] Graff K. F. Wave motion in elastic solids. Courier Corporation, 1975.

[H99] Hitrik, M. Bounds on scattering poles in one dimension, Commun. Math. Phys. 208(1999), 381-411.

[HLO06] Hoppe, J., Laptev, A., Östensson, J. Solitons and the removal of eigenvalues for fourth-order differential operators, Int. Math. Res. Not., (2006), 14 pp.

[I88] Iwasaki, K. Scattering Theory for 4-th Order Differential Operators, I. Japanese journal of mathematics. New series, 14(1) (1988), 1-57. 
[Iw88] Iwasaki, K. Scattering theory for 4-th order differential operator, II. Japanese journal of mathematics. New series, 14(1) (1988), 59-96.

[IK14] Iantchenko, A., Korotyaev, E. Resonances for 1D massless Dirac operators. Journal of Differential Equation, 256(8) (2014), 3038-3066.

[K03] Korotyaev, E. Characterization of the spectrum of Schrödinger operators with periodic distributions. Int. Math. Res. Not., 37 (2003), 2019-2031.

[K04] Korotyaev, E. Inverse resonance scattering on the half line. Asymptot. Anal. 37 (2004), no. 3-4, 215-226.

[K04x] Korotyaev, E. Stability for inverse resonance problem. Int. Math. Res. Not. 2004, no. 73, 3927-3936.

[K05] Korotyaev, E. Inverse resonance scattering on the real line. Inverse Problems 21.1 (2005), 325-341.

[K11] Korotyaev, E. Resonance theory for perturbed Hill operator, Asymp. Anal. 74 (2011), No 3-4, $199-227$.

[K14] Korotyaev E. Global estimates of resonances for 1D Dirac operators, Letters in Mathematical Physics, 104 (2014), No 1, 43-53.

[K16] Korotyaev, E. Resonances of third order differential operators. ArXiv preprint arXiv:1605.01842 (2016).

[K16x] Korotyaev E. Resonances for 1d Stark operators, to be published in Journal of Spectral Theory.

[K16xx] Korotyaev, E. Estimates of 1D resonances in terms of potentials, Journal d'Analyse Mathematique, 130 (2016), Issue 1, 151-166.

[Le96] Levin, B. Ya. Lectures on entire functions. Vol. 150. American Mathematical Soc., 1996.

[MSW10] Marletta, M.; Shterenberg, R.; Weikard, R., On the Inverse Resonance Problem for Schrödinger Operators, Commun. Math. Phys., 295(2010), 465-484.

[N07] Nedelec, N. Asymptotics of resonances for a Schrödinger operator with vector values. Journal of Functional Analysis, 244 (2007), 387-398.

[RS72] Reed, M., Simon, B. Methods of modern mathematical physics. vol. 1. Functional analysis, Academic Press, New York, 1972.

[RS75] Reed, M., Simon, B. Methods of modern mathematical physics. II. Fourier analysis, self-adjointness, Academic Press, New York, 1975.

[RS79] Reed, M.; Simon, B. Methods of Modern Mathematical Physics, Vol. III: Scattering Theory, Academic Press, New York, 1979.

[RS78] Reed, M.; Simon, B. Methods of Modern Mathematical Physics, Vol.IV: Analysis of Operators, Academic Press, New York, 1978.

[S00] Simon, B. Resonances in one dimension and Fredholm determinants, J. Funct. Anal. 178 (2000), no. 2, $396-420$.

[S05] Simon, B. Trace ideals and their applications. Second edition. Mathematical Surveys and Monographs, 120. AMS, Providence, RI, 2005.

[SZ91] Sjöstrand, J.; Zworski, M. Complex scaling and the distribution of scattering poles. J. Amer. Math. Soc. 4 (1991), no. 4, 729-769.

[TW59] Timoshenko, S. P., Woinowsky-Krieger, S. Theory of plates and shells. McGraw-hill, 1959.

[Z87] Zworski, M. Distribution of poles for scattering on the real line, J. Funct. Anal. 73(1987), $277-296$.

[Z02] Zworski, M. SIAM, J. Math. Analysis. A remark on isopolar potentials. 82(6) (2002), 1823-1826.

Saint-Petersburg State University, Universitetskaya nab. 7/9, St. Petersburg, 199034 RussiA, AN.BADANIN@GMAIL.COM, A.BADANIN@SPBU.RU, KOROTYAEV@GMAIL.COM, E.KOROTYAEV@SPBU.RU 